\title{
Optimum Shape Design of Composite Structures Using Boundary-Element Method
}

\section{DOI:}

$10.2514 / 1.11502$

\section{Document Version}

Accepted author manuscript

Link to publication record in Manchester Research Explorer

\section{Citation for published version (APA):}

Tafreshi, A. (2005). Optimum Shape Design of Composite Structures Using Boundary-Element Method. AIAA Journal, 43(6), 1349-1359. https://doi.org/10.2514/1.11502

\section{Published in:}

AIAA Journal

\section{Citing this paper}

Please note that where the full-text provided on Manchester Research Explorer is the Author Accepted Manuscript or Proof version this may differ from the final Published version. If citing, it is advised that you check and use the publisher's definitive version.

\section{General rights}

Copyright and moral rights for the publications made accessible in the Research Explorer are retained by the authors and/or other copyright owners and it is a condition of accessing publications that users recognise and abide by the legal requirements associated with these rights.

\section{Takedown policy}

If you believe that this document breaches copyright please refer to the University of Manchester's Takedown Procedures [http://man.ac.uk/04Y6Bo] or contact uml.scholarlycommunications@manchester.ac.uk providing relevant details, so we can investigate your claim.

\section{OPEN ACCESS}




\title{
OPTIMUM SHAPE DESIGN OF COMPOSITE STRUCTURES USING BOUNDARY -ELEMENT METHOD
}

\author{
Azam Tafreshi $\quad$ School of Mechanical, Aerospace and Civil Engineering (MACE) \\ The University of Manchester, Manchester \\ England, M60 1QD, United Kingdom \\ azam.tafreshi@manchester.ac.uk
}

\begin{abstract}
The boundary element method, combined with a numerical optimisation algorithm, has been employed for the shape optimisation of two-dimensional anisotropic structures. To find the optimum shape of a structure with the highest stiffness, the elastic compliance of the structure has been minimized subject to constraints upon stresses, weight and geometry. The optimum shapes of a series of anisotropic structures are obtained for maximum stiffness and minimum weight and stress, for specified loading conditions. The results are compared with the optimum shapes which were already created by the minimization of the structural weight while satisfying certain constraints upon stresses and geometry. A directly differentiated form of boundary integral equation with respect to geometric design variables is used to calculate shape design sensitivities of anisotropic materials. Because of the nonlinear nature of the mean compliance, weight and stresses, the numerical optimisation algorithm used is the feasible direction method, together with the golden section method for the one-dimensional search. Hermitian cubic spline functions are used to represent boundary shapes which offer considerable advantages in fitting a wide range of curves, and in the automatic remeshing process. Five example problems with anisotropic material properties are presented to demonstrate the applications of this general-purpose program.
\end{abstract}


KEY WORDS: $\quad$ Shape optimisation, boundary element method, composites, design sensitivity analysis, anisotropic materials, minimum compliance, weight minimization

\section{NOTATION}

$A_{j k}$

$\mathrm{a}_{\mathrm{mn}}$

$C_{j k}(P)$

$\mathrm{D}_{\mathrm{s}}(\mathrm{s}=1,4) \quad$ operator

$E_{k} \quad$ Young's modulus in the $\mathrm{x}_{\mathrm{k}}$ direction

$\mathrm{E}_{\mathrm{s}} \quad$ elastic compliance

$\left(\mathrm{E}_{\mathrm{s}}\right)_{0} \quad$ elastic compliance at initial step

$\mathrm{F}$

$\mathrm{F}_{0}$

$\mathrm{G}_{12}$

$\mathrm{g}_{\mathrm{j}}(\mathrm{X})$

$J(\zeta)$

$\mathrm{m}_{1 \mathrm{k}}, \mathrm{m}_{2 \mathrm{k}}$

$N^{c}(\zeta)$

$n_{1}, n_{2}$

$\mathrm{P}$

Q

complex constants

elastic compliance matrix

objective function

objective function at initial step

shear modulus

inequality constraints

Element

elastic body limiting value of the surface integral of $T_{j k}(p, Q)$

Jacobian of transformation from global Cartesian coordinates

to intrinsic coordinates of the element

unit vectors tangent and normal to the surface

quadratic shape function corresponding to the $c$ th node of the

direction cosines of the unit outward normal vector to the surface of the

load point at the surface of the elastic domain

field point at the surface of the elastic domain 
iteration number

$\left(\mathrm{R}_{\mathrm{i}}, \theta_{\mathrm{i}}\right) \quad$ polar coordinates

$\mathrm{r}_{\mathrm{jk}} \quad$ complex constants

$S^{\mathrm{q}}$

search direction at iteration $\mathrm{q}$

S

domain boundary

$\mathrm{S}_{\mathrm{b}} \quad$ bth element of the discretized boundary

$T_{j k}(P, Q) \quad j$ th component of the traction vector at point $\mathrm{Q}$ due

to a unit point load in the $k$ th direction at $\mathrm{P}$

$t_{j} \quad$ traction vector

$U_{j k} \quad j$ th component of the displacement vector at point Q due

to a unit point load in the kth direction at $\mathrm{P}$

$u_{j} \quad$ displacement vector

W weight

$\mathrm{W}_{0} \quad$ weight at initial step

X $\quad X_{i}=\left[X_{1}, X_{2}, \ldots, X_{n}\right]^{T} ;$ design variable vector

$x_{i} \quad$ rectangular Cartesian coordinates

$\bar{x}_{1}, \bar{x}_{2} \quad$ local coordiantes on an element

$\mathrm{Z}_{\mathrm{j}} \quad$ complex coordinates

$\alpha \quad$ scalar multiplier in steplength procedure

$\alpha_{\mathrm{j}}, \beta_{\mathrm{j}} \quad$ real constants

$\beta \quad$ scalar quantity that ensures the search direction will point into the feasible

zone

$\delta_{j k} \quad$ Kronecker delta 


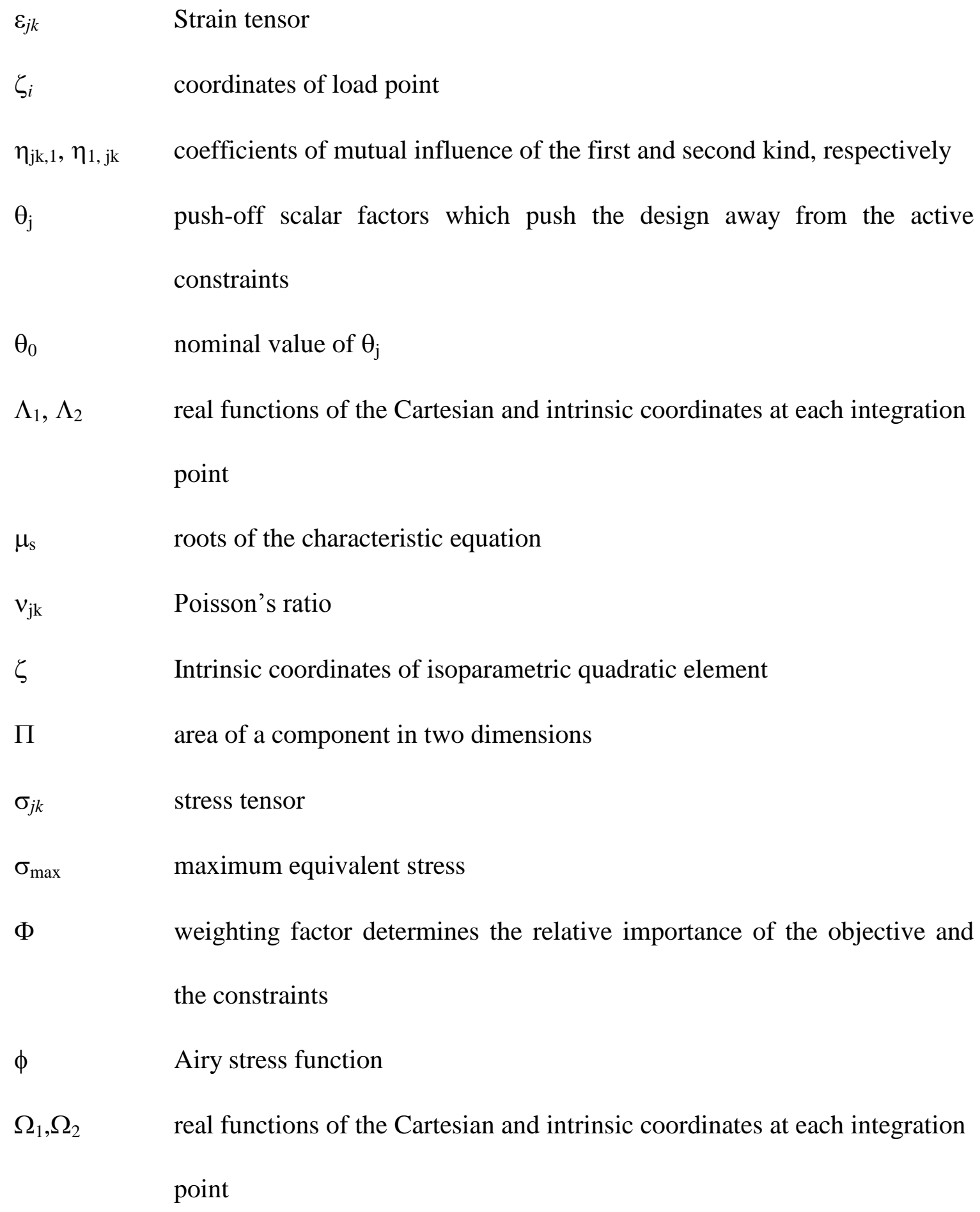

\section{INTRODUCTION}

Laminated composites are gaining importance in aircraft structural applications due to their attractive performance characteristics, for example, high strength-to-weight ratio, high stiffness-to-weight ratio, superior fatigue properties, and high corrosion resistance. The 
phenomenon of progressive failure in laminated composite structures is yet to be understood, and as a result, reliable strategies for designing optimal composite structures for desired life and strength are in demand. Up to now, shape optimization of composites has drawn little attention. However, the analytical formulation of two- and three-dimensional anisotropic elasticity, using the finite element method or boundary element method, has been well developed in the last three decades. This paper discusses the computational shape optimisation of anisotropic structures using the boundary element method, coupled with an optimisation algorithm.

Shape optimisation is an important area of current development in mechanical and structural design. Computerized procedures using optimisation algorithms can iteratively determine the optimum shape of a component while satisfying some objectives, without at the same time violating the design constraints.

There are three distinct classes of shape optimisation problems. In order of computational complexity, these are: size, shape, and topological optimisation. Size optimisation refers to the determination of specific geometric dimensions for a pre-selected design, such as the thickness of a shell, the size of a truss member, etc. This class of problems has been under investigation for decades. Shape optimisation introduces additional design variables, which allow for boundary movement. Due to its increased difficulty relative to size optimisation, the geometrical changes have been limited. However, it has gained importance in the aircraft and automotive industries, as well as others, providing improvements to turbines, airfoil shapes and connecting arms. Topological optimisation involves topological as well as shape and size modifications. In the case of discrete structures such as trusses or frames, 
topology is described by the number of bars and joints and the order in which the bars are connected to one another. For two- or three-dimensional continuum structures, the topological design variables may be the number of holes/cavities or may describe the connectivity of the domain such that the structure is simply or multiply-connected. In its simplest form, the topology optimization method solves the problem of distributing a given amount of material in a design domain subject to load and support conditions, such that the stiffness of the structure is maximized. Therefore, topology optimisation is essentially the optimum distribution of the material within the structure.

Although interest in and investigation of shape optimisation research has been intensifying during the past few decades, there is much work to be done before this class of optimisation can become an integral part of the design process. Design sensitivity analysis, that is the calculation of quantitative information on how the response of a structure is affected by changes in the design variables that define its shape, is a fundamental requirement for shape optimisation.

In a project sponsored by the UKAEA two general purpose computer programs using the BEM [1-4] for shape optimisation of isotropic structures; weight minimization and maximum stress minimization, respectively, were developed. In 1990 [1] it represented a novel application of the boundary element method to practical design optimisation problems and showed great potential for further development in the field of design optimisation.

The BEM being a boundary oriented technique can overcome a number of the difficulties associated with its main rival, the FEM. In respect of the continuously changing geometry, 
the accuracy of the FE analysis using an initial mesh of elements may become inadequate during the optimisation process. If during the optimisation process, the finite element mesh has to be re-generated, the cost is relatively high. The sensitivity analysis in the calculation of the derivatives with respect to the design variables may be obtained directly [1] in the boundary element approach rather than by approximate methods such as finite difference schemes.

In a recent study by the author [5], a directly differentiated form of the boundary integral equation with respect to geometric design variables was used to calculate stress and displacement derivatives for $2 \mathrm{D}$ anisotropic structures. The accuracy was compared against the results of the finite difference applied to the boundary element analysis. Not surprisingly, results obtained by analytical differentiation are much more accurate. In another study by the author [6], the weight minimization of anisotropic structures with stress constraints using the BEM is presented. The design sensitivity analysis using the BEM was combined with an optimisation algorithm to form an optimum shape design program for anisotropic structures. Different materials were analysed to investigate the effect of engineering constants on the optimum shape design of the components. It should be noted that to the author's knowledge, no other publications are available on the shape optimisation of composite materials using the boundary element method.

The objective of this work is directed towards the optimal shape design of an anisotropic elastic body which is of maximum stiffness and minimum weight under specified loadings, using the boundary element method. The elastic compliance of the structure will be minimized while there are constraints on the maximum stress and weight of the 
structure. To demonstrate the effectiveness of the procedure, a series of design problems will be analysed and discussed in detail. The results will be compared with those which were obtained with just the minimization of weight subject to stress and geometrical constraints [6].

\section{CONSTITUTIVE EQUATIONS FOR PLANE ANISOTROPIC ELASTICITY}

The stress-strain relations for a two-dimensional homogeneous, anisotropic elastic body in plane stress is [7]

$$
\left[\begin{array}{c}
\varepsilon_{11} \\
\varepsilon_{22} \\
2 \varepsilon_{12}
\end{array}\right]=\left[\begin{array}{lll}
a_{11} & a_{12} & a_{16} \\
a_{12} & a_{22} & a_{26} \\
a_{16} & a_{26} & a_{66}
\end{array}\right]\left[\begin{array}{c}
\sigma_{11} \\
\sigma_{22} \\
\sigma_{12}
\end{array}\right]
$$

where $\sigma_{\mathrm{jk}}$ and $\varepsilon_{\mathrm{jk}}(\mathrm{j}, \mathrm{k}=1,2)$, are the stresses and strains, respectively, and the coefficients $\mathrm{a}_{\mathrm{mn}}$ are the elastic compliances of the material. These compliances can be written in terms of engineering constants as

$$
\begin{aligned}
& a_{11}=\frac{1}{E_{1}} \quad a_{12}=-\frac{v_{12}}{E_{1}}=-\frac{v_{21}}{E_{2}} \quad a_{16}=\frac{\eta_{12,1}}{E_{1}}=\frac{\eta_{1,12}}{G_{12}} \\
& a_{22}=\frac{1}{E_{2}} \quad a_{26}=\frac{\eta_{12,2}}{E_{2}}=\frac{\eta_{2,12}}{G_{12}} \quad a_{66}=\frac{1}{G_{12}}
\end{aligned}
$$

where $\mathrm{G}_{12}$ is the shear modulus in the $\mathrm{x}_{1}-\mathrm{x}_{2}$ plane. The quantities $\eta_{\mathrm{jk}, 1}, \eta_{1, \mathrm{jk}}$ are referred to by Lekhnitskii [8] as the coefficients of mutual influence of the first and second kind, respectively. For specially orthotropic materials, $a_{16}=a_{26}=0$. [8].

The compatibility equation of strains is

$$
\frac{\partial^{2} \varepsilon_{11}}{\partial x_{2}{ }^{2}}+\frac{\partial^{2} \varepsilon_{22}}{\partial x_{1}{ }^{2}}=2 \frac{\partial^{2} \varepsilon_{12}}{\partial x_{1} \partial x_{2}}
$$


and equilibrium is satisfied by taking stresses in terms of derivatives of the Airy stress function $\phi\left(\mathrm{x}_{1}, \mathrm{x}_{2}\right)$ as

$$
\sigma_{11}=\frac{\partial^{2} \phi}{\partial x_{2}^{2}} \quad \sigma_{22}=\frac{\partial^{2} \phi}{\partial x_{1}^{2}} \quad \sigma_{12}=-\frac{\partial^{2} \phi}{\partial x_{1} \partial x_{2}}
$$

Combining equations 1,3 and 4 , the governing equation for the two dimensional problem of anisotropic elasticity can be obtained

$a_{22} \frac{\partial^{4} \phi}{\partial x_{1}^{4}}-2 a_{26} \frac{\partial^{4} \phi}{\partial x_{1}^{3} \partial x_{2}}+\left(2 a_{12}+a_{66}\right) \frac{\partial^{4} \phi}{\partial x_{1}^{2} \partial x_{2}^{2}}-2 a_{16} \frac{\partial^{4} \phi}{\partial x_{1} \partial x_{2}^{3}}+a_{66} \frac{\partial^{4} \phi}{\partial x_{2}^{4}}=0$.

By introducing the operator $\mathrm{D}_{\mathrm{s}}(\mathrm{s}=1,4)$ as

$D_{s}=\frac{\partial}{\partial x_{2}}-\mu_{s} \frac{\partial}{\partial x_{1}}$

Equation (5) becomes

$D_{1} D_{2} D_{3} D_{4}(\phi)=0$

and $\mu_{\mathrm{s}}$ are the four roots of the characteristic equation

$$
\left[a_{22}-2 \mu a_{26}+\left(2 a_{12}+a_{66}\right) \mu^{2}-2 a_{16} \mu^{3}+a_{11} \mu^{4}\right] \frac{d^{4} \phi}{d z^{4}}=0
$$

To have a solution for the stress function, the term in square brackets must be zero. Leknitskii [8] has shown that, for an anisotropic material, these roots are distinct and must be either purely imaginary or complex and they may be denoted by

$$
\mu_{1}=\alpha_{1}+i \beta_{1} \quad, \quad \mu_{2}=\alpha_{2}+i \beta_{2} \quad, \quad \mu_{3}=\bar{\mu}_{1} \quad, \quad \mu_{4}=\bar{\mu}_{2}
$$

where $\alpha_{\mathrm{j}}$ and $\beta_{\mathrm{j}},(\mathrm{j}=1,2)$, are real constants, $i=\sqrt{-1}$ and the overbar represents the complex conjugate. The characteristic directions may thus be denoted by

$$
z_{j}=x_{1}+\mu_{j} x_{2} \quad j=1,2
$$


and their complex conjugates.

\section{BOUNDARY ELEMENT METHOD FOR ANISOTROPIC MATERIALS}

The boundary element method is based on the unit load solutions in an infinite body, known as the fundamental solutions, used with the reciprocal work theorem and appropriate limit operations. The boundary integral equation in the direct formulation of the BEM for anisotropic materials is an integral constraint equation relating the tractions $\left(\mathrm{t}_{\mathrm{j}}\right)$ and the displacements $\left(\mathrm{u}_{\mathrm{j}}\right)$ at the boundary $\mathrm{s}$ of the homogeneous elastic domain, and it can be written as

$C_{j k} u_{j}(P)+\int_{s} T_{j k}(P, Q) u_{j}(Q) d s(Q)=\int_{s} U_{j k}(P, Q) t_{j}(Q) d s(Q) \quad j, k=1,2$

where $\mathrm{P}\left(\zeta_{1}, \zeta_{2}\right)$ and $\mathrm{Q}\left(\mathrm{x}_{1}, \mathrm{x}_{2}\right)$ are the load and field points, respectively. $U_{j k}(\mathrm{P}, \mathrm{Q})$ and $T_{j k}$ $(\mathrm{P}, \mathrm{Q})$ are the fundamental solutions that represent the displacements and tractions, respectively, in the $\mathrm{x}_{\mathrm{k}}$ direction at $\mathrm{Q}$ because of a unit load in the $\mathrm{x}_{\mathrm{j}}$ direction at $\mathrm{P}$ in an infinite anisotropic body. The constant $\mathrm{C}_{\mathrm{jk}}$ depends on the local geometry of the boundary at $\mathrm{P}$, whether it lies on a smooth surface or a sharp corner. In terms of generalised complex variables

$$
\begin{aligned}
& z_{1}=\left(x_{1}-\zeta_{1}\right)+\mu_{1}\left(x_{2}-\zeta_{2}\right) \\
& z_{2}=\left(x_{1}-\zeta_{1}\right)+\mu_{2}\left(x_{2}-\zeta_{2}\right)
\end{aligned}
$$

the fundamental solution for displacements and tractions, respectively, are as follows:

$$
\begin{aligned}
& U_{j k}=2 \operatorname{Re}\left[r_{k 1} A_{j 1} \ln \left(z_{1}\right)+r_{k 2} A_{j 2} \ln \left(z_{2}\right)\right] \\
& T_{j 1}=2 n_{1} \operatorname{Re}\left[\mu_{1}^{2} A_{j 1} / z_{1}+\mu_{2}^{2} A_{j 2} / z_{2}\right]-2 n_{2} \operatorname{Re}\left[\mu_{1} A_{j 1} / z_{1}+\mu_{2} A_{j 2} / z_{2}\right] \\
& T_{j 2}=-2 n_{1} \operatorname{Re}\left[\mu_{1} A_{j 1} / z_{1}+\mu_{2} A_{j 2} / z_{2}\right]+2 n_{2} \operatorname{Re}\left[A_{j 1} / z_{1}+A_{j 2} / z_{2}\right]
\end{aligned}
$$

where $\mathrm{n}_{\mathrm{j}}$ are the unit outward normal components at $\mathrm{Q}$ with respect to the $\mathrm{x}_{1}-\mathrm{x}_{2}$ coordinate system. The constants $r_{\mathrm{kj}}$ are 


$$
\begin{aligned}
& r_{1 j}=a_{11} \mu_{j}^{2}+a_{12}-a_{16} \mu_{j} \\
& r_{2 j}=a_{12} \mu_{j}+a_{22} / \mu_{j}-a_{26}
\end{aligned}
$$

and $\mathrm{A}_{\mathrm{jk}}$ are complex constants that can be obtained from the following set of equations:

$$
\begin{aligned}
& A_{j 1}-\bar{A}_{j 1}+A_{j 2}-\bar{A}_{j 2}=\delta_{j 2} / 2 \pi i \\
& \mu_{1} A_{j 1}-\bar{\mu}_{1} \bar{A}_{j 1}+\mu_{2} A_{j 2}-\bar{\mu}_{2} \bar{A}_{j 2}=-\delta_{j 1} / 2 \pi i \\
& r_{11} A_{j 1}-\bar{r}_{11} \bar{A}_{j 1}+r_{21} A_{j 2}-\bar{r}_{21} \bar{A}_{j 2}=0 . \\
& r_{12} A_{j 1}-\bar{r}_{12} \bar{A}_{j 1}+r_{22} A_{j 2}-\bar{r}_{22} \bar{A}_{j 2}=0 .
\end{aligned}
$$

For the details of these, the reader is referred to references [9-12].

The boundary element implementation of equation 11 entails boundary discretization. Quadratic isoparametric elements are chosen for the analyses. Substitution of these isoparametric representations into equation 11 will result in a set of linear algebraic equations for the unknown displacements and tractions at the nodes on the boundary of the solution domain as follows

$$
\mathrm{AU}=\mathrm{B}
$$

where $A$ is the final coefficient matrix, $\mathrm{U}$ is the vector containing the unknown variables (displacements or tractions), and the second member B is a vector. A and B result after the rearrangement of the system of equations obtained from the substitution of the boundary conditions in the discretized version of equation 11. These linear algebraic equations can then be solved by standard matrix solution techniques.

To calculate surface stresses from the already calculated surface tractions and displacements, it is necessary to consider a local system of coordinates $\left(\mathrm{x}_{1}, \mathrm{x}_{2}\right)$. Let $\mathrm{m}_{1 \mathrm{k}}$ be the unit vector in direction tangential to the surface and $\mathrm{m}_{2 \mathrm{k}}$ the one in the direction normal 
to the surface. Let $\mathrm{u}_{\mathrm{j}}, \mathrm{t}_{\mathrm{j}}, \varepsilon_{\mathrm{jk}}$, and $\sigma_{\mathrm{jk}}$ be the displacements, tractions, strains, and stresses, respectively, in the local coordinates. The displacement in the tangential direction is

$$
\bar{u}_{j}(\zeta)=N^{c}(\zeta) u_{j}^{c} m_{1 k}
$$

Where $\mathrm{N}^{\mathrm{c}}(\zeta)(\mathrm{c}=1,2,3)$ are the quadratic shape functions of intrinsic coordinates. The strain in the tangential direction is obtained by this expression to give

$$
\bar{\varepsilon}_{11}(\zeta)=\frac{d N^{c}(\zeta)}{d \zeta} u_{j}^{c} m_{1 k} \frac{1}{J(\zeta)}
$$

Then, using the constitutive equation (Eq. 1), the components of the stress tensor in the local coordinate system can be calculated, and by a simple transformation the stress components in the global system can be obtained.

\section{SHAPE DESIGN SENSITIVITY ANALYSIS OF 2D ANISOTROPIC MATERIALS}

Implicit differentiation of the BIE equation with respect to a design variable, $\mathrm{x}_{h}(h=1,2)$ (which is most likely to be the coordinate of a node on the movable part of the boundary) results in the following equation

$$
\begin{aligned}
& C_{j k}(P) \frac{\partial u_{j}(P)}{\partial x_{h}}+\frac{\partial C_{j k}}{\partial x_{h}} u_{j}(P)+\int_{s}\left(\frac{\partial T_{j k}(P, Q)}{\partial x_{h}} u_{j}(Q)+T_{j k}(P, Q) \frac{\partial u_{j}(Q)}{\partial x_{h}}\right) d s(Q)+ \\
& \int_{s} T_{j k}(P, Q) u_{j}(Q) \frac{\partial(d s(Q))}{\partial x_{h}}=\int_{s}\left(\frac{\partial U_{j k}(P, Q)}{\partial x_{h}} t_{j}(Q)+U_{j k}(P, Q) \frac{\partial t_{j}(Q)}{\partial x_{h}}\right) d s(Q)+ \\
& \int_{s} U_{j k}(P, Q) t_{j}(Q) \frac{\partial(d s(Q))}{\partial x_{h}}
\end{aligned}
$$


The derivatives of the terms which only depend on the geometry will be carried out similar to the isotropic materials [1-4]. The derivatives of the remaining terms such as $\mathrm{U}_{\mathrm{jk}}$ and $\mathrm{T}_{\mathrm{jk}}$ for anisotropic materials will be as follows:

$$
\begin{aligned}
& \frac{\partial U_{j k}}{\partial x_{h}}=2 \frac{\partial}{\partial x_{h}}\left[\operatorname{Re}\left(r_{k 1} A_{j 1} \ln \left(z_{1}\right)+r_{k 2} A_{j 2} \ln \left(z_{2}\right)\right)\right] \\
& \frac{\partial T_{j 1}}{\partial x_{h}}=2 n_{1} \frac{\partial}{\partial x_{h}}\left[\operatorname{Re}\left(\frac{\mu_{1}^{2} A_{j 1}}{z_{1}}+\frac{\mu_{2}^{2} A_{j 2}}{z_{2}}\right)\right]+2 \operatorname{Re}\left(\frac{\mu_{1}^{2} A_{j 1}}{z_{1}}+\frac{\mu_{2}^{2} A_{j 2}}{z_{2}}\right) \frac{\partial\left(n_{1}\right)}{\partial x_{h}} \\
& -2 n_{2} \frac{\partial}{\partial x_{h}}\left[\operatorname{Re}\left(\frac{\mu_{1} A_{j 1}}{z_{1}}+\frac{\mu_{2} A_{j 2}}{z_{2}}\right)\right]-2 \operatorname{Re}\left(\frac{\mu_{1} A_{j 1}}{z_{1}}+\frac{\mu_{2} A_{j 2}}{z_{2}}\right) \frac{\partial\left(n_{2}\right)}{\partial x_{h}} \\
& \frac{\partial T_{j 2}}{\partial x_{h}}=-2 n_{1} \frac{\partial}{\partial x_{h}}\left[\operatorname{Re}\left(\frac{\mu_{1} A_{j 1}}{z_{1}}+\frac{\mu_{2} A_{j 2}}{z_{2}}\right)\right]-2 \operatorname{Re}\left(\frac{\mu_{1} A_{j 1}}{z_{1}}+\frac{\mu_{2} A_{j 2}}{z_{2}}\right) \frac{\partial\left(n_{1}\right)}{\partial x_{h}} \\
& +2 n_{2} \frac{\partial}{\partial x_{h}}\left[\operatorname{Re}\left(\frac{A_{j 1}}{z_{1}}+\frac{A_{j 2}}{z_{2}}\right)\right]+2 \operatorname{Re}\left(\frac{A_{j 1}}{z_{1}}+\frac{A_{j 2}}{z_{2}}\right) \frac{\partial\left(n_{2}\right)}{\partial x_{h}}
\end{aligned}
$$

where the coefficients $\mu_{\mathrm{j}}$ and $\mathrm{A}_{\mathrm{jk}}$ depend on the material properties and are independent of the design variables. To calculate the above derivatives, the complex values, $\ln \left(z_{j}\right)$ and

$$
\begin{aligned}
& \frac{1}{z_{j}} \text {, can be written as } \\
& \ln \left(z_{j}\right)=\ln \left|z_{j}\right|+i \arg \left(z_{j}\right), \frac{1}{z_{j}}=\frac{\bar{z}_{j}}{\left|z_{j}\right|^{2}}
\end{aligned}
$$

Defining the real functions $\Lambda_{\mathrm{j}}$ and $\Omega_{\mathrm{j}}$ as

$$
\begin{aligned}
& \Lambda_{1}=\left(x_{1}-\zeta_{1}\right)+\alpha_{1}\left(x_{2}-\zeta_{2}\right) \\
& \Lambda_{2}=\left(x_{1}-\zeta_{1}\right)+\alpha_{2}\left(x_{2}-\zeta_{2}\right) \\
& \Omega_{1}=-\beta_{1} \zeta_{2}+\beta_{1} x_{2} \\
& \Omega_{2}=-\beta_{2} \zeta_{2}+\beta_{2} x_{2}
\end{aligned}
$$


the complex coordinates and their conjugates can be written as

$$
z_{j}=\Lambda_{j}+i \Omega_{j}, \bar{z}_{j}=\Lambda_{j}-i \Omega_{j}
$$

By substituting equations 21-23 into equations 20 , the derivatives of the kernel products with respect to the design variable $\mathrm{x}_{l}$ can be obtained.

$$
\begin{aligned}
& \frac{\partial U_{j k}}{\partial x_{h}}=2 \operatorname{Re}\left(r_{k 1} A_{j 1}\right) \frac{\partial}{\partial x_{h}}\left[\ln \left|z_{1}\right|\right]+2 \operatorname{Re}\left(r_{k 1} A_{j 1} i\right) \frac{\partial}{\partial x_{h}}\left[\arg \left(z_{1}\right)\right] \\
& +2 \operatorname{Re}\left(r_{k 2} A_{j 2}\right) \frac{\partial}{\partial x_{h}}\left[\ln \left|z_{2}\right|\right]+2 \operatorname{Re}\left(r_{k 2} A_{j 2} i\right) \frac{\partial}{\partial x_{h}}\left[\arg \left(z_{2}\right)\right]
\end{aligned}
$$

$$
\begin{aligned}
& \frac{\partial T_{j 1}}{\partial x_{h}}=2 \operatorname{Re}\left[\frac{\mu_{1}^{2} A_{j 1}}{z_{1}}+\frac{\mu_{2}^{2} A_{j 2}}{z_{2}}\right] \frac{\partial n_{1}}{\partial x_{h}}-2 \operatorname{Re}\left[\frac{\mu_{1} A_{j 1}}{z_{1}}+\frac{\mu_{2} A_{j 2}}{z_{2}}\right] \frac{\partial n_{2}}{\partial x_{h}} \\
& +2 n_{1} \operatorname{Re}\left(\mu_{1}^{2} A_{j 1}\right) \frac{\partial}{\partial x_{h}}\left(\frac{\Lambda_{1}}{\left|z_{1}\right|^{2}}\right)+2 n_{1} \operatorname{Re}\left(-\mu_{1}^{2} A_{j 1} i\right) \frac{\partial}{\partial x_{h}}\left(\frac{\Omega_{1}}{\left|z_{1}\right|^{2}}\right)+2 n_{1} \operatorname{Re}\left(\mu_{2}^{2} A_{j 2}\right) \frac{\partial}{\partial x_{h}}\left(\frac{\Lambda_{2}}{\left|z_{2}\right|^{2}}\right) \\
& +2 n_{1} \operatorname{Re}\left(-\mu_{2}^{2} A_{j 2} i\right) \frac{\partial}{\partial x_{h}}\left(\frac{\Omega_{2}}{\left|z_{2}\right|^{2}}\right)-2 n_{2} \operatorname{Re}\left(\mu_{1} A_{j 1}\right) \frac{\partial}{\partial x_{h}}\left(\frac{\Lambda_{1}}{\left|z_{1}\right|^{2}}\right)-2 n_{2} \operatorname{Re}\left(-\mu_{1} A_{j 1} i\right) \frac{\partial}{\partial x_{h}}\left(\frac{\Omega_{1}}{\left|z_{1}\right|^{2}}\right) \\
& -2 n_{2} \operatorname{Re}\left(\mu_{2} A_{j 2}\right) \frac{\partial}{\partial x_{h}}\left(\frac{\Lambda_{2}}{\left|z_{2}\right|^{2}}\right)-2 n_{2} \operatorname{Re}\left(-\mu_{2} A_{j 2} i\right) \frac{\partial}{\partial x_{h}}\left(\frac{\Omega_{2}}{\left|z_{2}\right|^{2}}\right)
\end{aligned}
$$




$$
\begin{aligned}
& \frac{\partial T_{j 2}}{\partial x_{h}}=-2 \operatorname{Re}\left[\frac{\mu_{1} A_{j 1}}{z_{1}}+\frac{\mu_{2} A_{j 2}}{z_{2}}\right] \frac{\partial n_{1}}{\partial x_{h}}+2 \operatorname{Re}\left[\frac{A_{j 1}}{z_{1}}+\frac{A_{j 2}}{z_{2}}\right] \frac{\partial n_{2}}{\partial x_{h}} \\
& -2 n_{1} \operatorname{Re}\left(\mu_{1} A_{j 1}\right) \frac{\partial}{\partial x_{h}}\left(\frac{\Lambda_{1}}{\left|z_{1}\right|^{2}}\right)-2 n_{1} \operatorname{Re}\left(-i \mu_{1} A_{j 1}\right) \frac{\partial}{\partial x_{h}}\left(\frac{\Omega_{1}}{\left|z_{1}\right|^{2}}\right)-2 n_{1} \operatorname{Re}\left(\mu_{2} A_{j 2}\right) \frac{\partial}{\partial x_{h}}\left(\frac{\Lambda_{2}}{\left|z_{2}\right|^{2}}\right) \\
& -2 n_{1} \operatorname{Re}\left(-i \mu_{2} A_{j 2}\right) \frac{\partial}{\partial x_{h}}\left(\frac{\Omega_{2}}{\left|z_{2}\right|^{2}}\right)+2 n_{2} \operatorname{Re}\left[A_{j 1}\right] \frac{\partial}{\partial x_{h}}\left(\frac{\Lambda_{1}}{\left|z_{1}\right|^{2}}\right)+2 n_{2} \operatorname{Re}\left[-i A_{j 1}\right] \frac{\partial}{\partial x_{h}}\left(\frac{\Omega_{1}}{\left|z_{1}\right|^{2}}\right) \\
& +2 n_{2} \operatorname{Re}\left[A_{j 2}\right] \frac{\partial}{\partial x_{h}}\left(\frac{\Lambda_{2}}{\left|z_{2}\right|^{2}}\right)+2 n_{2} \operatorname{Re}\left(-i A_{j 2}\right) \frac{\partial}{\partial x_{h}}\left(\frac{\Omega_{2}}{\left|z_{2}\right|^{2}}\right)
\end{aligned}
$$

Therefore, the design sensitivity analysis is carried out by implicit differentiation of the structural response (equation 16) with respect to design variables $\mathrm{x}_{l}$, which are the coordinates of some nodes of the movable part of the boundary,

$$
\frac{\partial A}{\partial x_{h}} U+A \frac{\partial U}{\partial x_{h}}=\frac{\partial B}{\partial x_{h}} \quad \Rightarrow \quad A \frac{\partial U}{\partial x_{h}}=\left(\frac{\partial B}{\partial x_{h}}-\frac{\partial A}{\partial x_{h}} U\right)
$$

This is a set of linear algebraic equations for the unknown gradients, $\frac{\partial U}{\partial x_{h}}$, and equivalent to solving the same equation as 16. Thus, if the quantity in brackets in equation 27 is separately assembled, then the displacement derivative vector $\frac{\partial U}{\partial x_{h}}$ can be computed in one pass by re-entering the equation solver.

The gradients of stresses usually require the intermediate calculation of the gradients of displacements with respect to the design variables. For the derivatives of the stresses, both sides of equation (18) are differentiated and the gradients of strain in the tangential direction is obtained from 
$\frac{\partial \bar{\varepsilon}_{11}}{\partial x_{h}}=\frac{d N^{c}(\zeta)}{d \zeta} \frac{\partial u_{k}^{c}}{\partial x_{h}} m_{1 k} \frac{1}{J(\zeta)}+\frac{d N^{c}(\zeta)}{d \zeta} u_{k}^{c} \frac{\partial m_{1 k}}{\partial x_{h}} \frac{1}{J(\zeta)}+\frac{d N^{c}(\zeta)}{d \zeta} u_{k}^{c} m_{1 k} \frac{-1}{[J(\zeta)]^{2}} \frac{\partial[J(\zeta)]}{\partial x_{h}}$

which is a function of gradients of displacements previously calculated. The gradients of the stresses can then be determined by differentiating the constitutive equations for the anisotropic materials.

In reference [5], the derivatives of displacements and stresses with respect to design variables for anisotropic materials are calculated both by this direct analytical differentiation method and also by the finite difference method. The former is shown to be both more accurate and less time consuming.

\section{ANALYTIC CALCULATION OF WEIGHT AND ITS DERIVATIVES}

For a $2 \mathrm{D}$ elastic component with a uniform mass density and thickness, minimizing the area is equivalent to minimizing the structural weight. Here, uniform mass density for anisotropic materials has been assumed. As shown in references $[1,6]$ the area of the structure and its derivatives with respect to the design variables can also be determined using the BEM. Based on the Green's theorem, the area of the domain can be written as line integral over the boundary. Then by discretization of the domain to M quadratic isoparametric elements, the area of the domain can be obtained.

$$
\Pi=\frac{1}{2} \int_{s}\left(x_{1} d x_{2}-x_{2} d x_{1}\right)=\frac{1}{2} \sum_{b=1}^{M} \int_{s_{b}}\left[x_{1}(\zeta) n_{1}+x_{2}(\zeta) n_{2}\right] J(\zeta) d \zeta
$$

By differentiating the above equation with respect to the geometric design variable $\mathrm{x}_{\mathrm{h}}$, the weight derivatives can be obtained. 
If $\mathrm{x}_{h}$ is the $\mathrm{x}_{1}$ coordinate of movable node then

$$
\frac{\partial \prod}{\partial x_{1}}=\frac{1}{2} \sum_{b=1}^{M} \int\left[\frac{\partial x_{1}(\zeta)}{\partial x_{1}} \frac{d x_{2}}{d \zeta}-x_{2}(\zeta) \frac{\partial}{\partial x_{1}}\left(\frac{d x_{1}}{d \zeta}\right)\right] d \zeta
$$

If $\mathrm{x}_{h}$ is the $\mathrm{x}_{2}$ coordinate of movable node therefore,

$$
\frac{\partial \prod}{\partial x_{2}}=\frac{1}{2} \sum_{b=1}^{M} \int_{s_{b}}\left[x_{1}(\zeta) \frac{\partial}{\partial x_{2}}\left(\frac{d x_{2}}{d \zeta}\right)-\frac{\partial x_{2}(\zeta)}{\partial x_{2}}\left(\frac{d x_{1}}{d \zeta}\right)\right] d \zeta
$$

\section{ANALYTIC CALCULATION OF ELASTIC COMPLIANCE AND ITS DERIVATIVES}

The elastic compliance is evaluated as the strain energy of the structure

$$
E_{s}=\frac{1}{2} \int_{s} t_{j} u_{j} d s
$$

If the boundary of the structure is discretized to $\mathrm{M}$ quadaratic boundary elements, therefore, the elastic compliance can be evaluated as

$$
E_{s}=\frac{1}{2} \sum_{b=1}^{M} \int_{-1}^{1}\left[t_{j}^{c}(\zeta) N^{c}(\zeta)\right]\left[u_{j}^{d}(\zeta) N^{d}(\zeta)\right] J(\zeta) d \zeta
$$

The derivatives of the elastic compliance with respect to the design variable $\mathrm{x}_{\mathrm{h}}$ can also be calculated analytically as shown below

$$
\begin{aligned}
& \frac{\partial E_{s}}{\partial x_{h}}=\frac{1}{2} \sum_{b=1}^{M} \int\left[\frac{t_{j}^{c}(\zeta)}{d x_{h}} N^{c}(\zeta)+t_{j}^{c}(\zeta) \frac{d N^{c}(\zeta)}{d x_{h}}\right]\left[u_{j}^{d}(\zeta) N^{d}(\zeta)\right] J(\zeta) d \zeta+ \\
& \frac{1}{2} \sum_{b=1}^{M} \int_{s_{b}}\left[t_{j}^{c}(\zeta) N^{c}(\zeta)\right]\left[\frac{u_{j}^{d}(\zeta)}{d x_{h}} N^{d}(\zeta)+u_{j}^{d}(\zeta) \frac{d N^{d}(\zeta)}{d x_{h}}\right] J(\zeta) d \zeta+\frac{1}{2} \sum_{b=1}^{M} \int\left[t_{s_{b}}^{c}(\zeta) N^{c}(\zeta)\right]\left[u_{j}^{d}(\zeta) N^{d}(\zeta)\right] \frac{\partial J(\zeta)}{d x_{h}} d \zeta
\end{aligned}
$$


Equation (34) shows that in order to obtain the compliance derivatives, the derivatives of tractions and displacements must be already calculated. Therefore, during the analysis the compliance derivatives will be evaluated after the stresses, displacements and their derivatives are obtained.

A simple plate under tension is studied to demonstrate the validity of the approach. The dimensions and loading are shown in Fig.1. Due to the symmetry, only the right half of the plate is considered. The model contains 40 quadratic elements, four elements on each side $\mathrm{AC}$ and $\mathrm{ED}$ and 16 elements on each side $\mathrm{CD}$ and $\mathrm{AE}$. Five different anisotropic materials from Table 1 are selected, materials 1 to 5 . Material 1 is isotropic but in the current analysis it is assumed as if it is anisotropic $\left[\mathrm{E}_{1}=\mathrm{E}_{2}, \mathrm{G}_{12}=\mathrm{E}_{1} / 2\left(1+\mathrm{v}_{12}\right)\right]$. The design variable, the $\mathrm{x}$ coordinate of point $\mathrm{M}$, is selected to represent a change in the shape of the edge BC. Table 2 shows the derivatives of compliance of the plate with respect to the $\mathrm{x}$ coordinate of point $\mathrm{M}$ calculated by the boundary element method described above. These are compared with the results of the finite difference approach used with the boundary element analysis. For the finite difference results, different movements of the design variable, from $\mathrm{d}=10^{-2}$ to $\mathrm{d}=10^{-6}$ have been considered and the analysis was carried out with double precision. For the direct differentiation analysis single precision was applied. For the finite difference approach the step size $\mathrm{d}=10^{-7}$ gives the best results, but the agreement with the analytical results is still only moderately good. 
Tafreshi, A. June 2005 In : AIAA Journal, 43, 6, p. 1349-1359 11 p.

Table 1 Elastic properties of the selected materials

\begin{tabular}{|l|l|l|l|l|l|l|}
\hline Material & $\mathrm{E}_{11}(\mathrm{GPa})$ & $\mathrm{E}_{22}(\mathrm{GPa})$ & $v_{12}$ & $\mathrm{G}_{12}(\mathrm{GPa})$ & \multicolumn{2}{|l|}{ Complex parameters } \\
\hline 1 & & & & & $\mu_{1}$ & $\mu_{2}$ \\
\hline 2 & 210.9 & 210.9 & 0.29 & 81.8 & $\mathrm{i}$ & $\mathrm{i}$ \\
\hline 3 & 18.9 & 18.9 & 0.845 & 55.6 & $-0.915+0.403 \mathrm{i}$ & $0.915+0.403 \mathrm{i}$ \\
\hline 4 & 276.0 & 6.9 & 0.25 & 3.4 & $8.948 \mathrm{i}$ & $0.706 \mathrm{i}$ \\
\hline 5 & 148.07 & 11.08 & 0.295 & 6.40 & $4.683 \mathrm{i}$ & $0.780 \mathrm{i}$ \\
\hline
\end{tabular}

Table 2 Derivatives of the compliance

\begin{tabular}{|c|c|c|c|c|c|c|}
\hline $\begin{array}{l}\text { Material } \\
\text { No. }\end{array}$ & $\begin{array}{l}\text { Direct } \\
\text { differentiation } \\
\text { of the BIE }\end{array}$ & \multicolumn{5}{|c|}{$\begin{array}{l}\text { Finite difference method using the BEM analysis with } \\
\text { different step sizes }(d)\end{array}$} \\
\hline 1 & 0.2133 & 0.21141 & 0.23256 & 0.23466 & 0.23483 & 0.23438 \\
\hline 2 & 0.0153 & 0.01861 & 0.020478 & 0.02066 & 0.02069 & 0.02074 \\
\hline 3 & 0.0070 & 0.00695 & 0.00765 & 0.00772 & 0.00773 & 0.00772 \\
\hline 4 & 0.0112 & 0.01118 & 0.01230 & 0.01241 & 0.01242 & 0.01241 \\
\hline 5 & 0.0143 & 0.01412 & 0.015538 & 0.01567 & 0.01569 & 0.01566 \\
\hline
\end{tabular}




\section{PROBLEM STATEMENT AND METHOD OF OPTIMIZATION}

The shape optimisation problem can be stated mathematically as

$$
\begin{array}{ll}
\operatorname{Min}\left\{F\left(X_{1}\right\}\right. & 1=1,2,3, \ldots, n \\
\text { Subject to } g_{i}\left(X_{1}\right) \leq 0 . & i=1,2,3 \ldots \ldots, m \\
X_{1}^{L} \leq X_{1} \leq X_{1}^{U} &
\end{array}
$$

in which $\mathrm{g}_{\mathrm{i}}$ is the constraint function describing the ith structural response, $\mathrm{X}$ is a vector of design variables that defines the shape of the variable part of the structure, and $\mathrm{X}_{1}^{\mathrm{L}}$ and $\mathrm{X}_{1}^{\mathrm{U}}$ are the lower and the upper limits of the shape variables [13].

In a recent study by the author, an optimum shape design algorithm was developed by the coupling of an optimising technique with a boundary element stress analyser for stress minimization of 2D anisotropic structures [5]. Applications of this general purpose computer program to the optimum shape design of bars and holes in plates with anisotropic materials were presented. In this case the objective function is highly nonlinear, while the constraints are linear. The optimisation method used was the extended interior penalty function approach, and for unconstrained minimization, the BroydonFletcher-Goldfarb-Shanno variable metric method was employed. Hermitian cubic spline functions, which are combinations of piecewise cubic curves with continuous first and second derivatives at the ends of each curve, were used to represent the boundary shape. This provides a flexible and compact method for defining boundary geometry using a small number of design variables. They also reduce the need for optimisation constraints to avoid impractical design during the optimisation procedure [1-6]. 
In another study by the author [6] , an optimisation algorithm was developed to minimize the structural weight of a 2D anisotropic structure while satisfying certain constraints upon stresses and geometry. The boundary element method was used for the stress analysis and also for the shape sensitivity analysis. Since both the objective function and constraints are non-linear, the feasible direction method (FDM) was employed [1-4].

In the present study, to find the optimal shape of a structure for the highest stiffness and minimum weight, the elastic compliance has been minimized. The constraints are imposed on the maximum allowable stress and maximum weight. Therefore, the elastic compliance is treated as the objective function whereas stresses and weight are treated as the inequality constraints. The feasible direction method is employed as the optimisation algorithm.

\section{FEASIBLE DIRECTION METHOD}

The FDM was first proposed by Zoutendijk [14] and later Vanderplaats et al [15] applied the algorithm on design optimisation of space truss structures. Since then the FDM has been one of the most popular methods for finite element analysis based structural optimisation [13]. It is also shown that FDM can be successfully used for boundary element analysis based structural optimisation [1-6].

FDM deals directly with the nonlinearity of the problem. FDM is constructed to deal with inequality constraints, and for use in the case of equality constrained problems, those equality constraints can be easily converted to inequality constraints. 
FDM determines a usable-feasible direction in which the design point may be moved in the design space. This direction is 'feasible' because it does not violate, at least over an infinitely small step, any of the constraints; and 'usable' because it results in a reduction of the objective function. The basic idea is to choose a starting point satisfying all the constraints and to move to a better point according to the iterative scheme $\mathrm{X}^{\mathrm{q}}=\mathrm{X}^{\mathrm{q}-1}+\alpha_{\mathrm{q}} \mathrm{S}^{\mathrm{q}}$

where $S^{q}$ is the vector of search direction and $\alpha_{q}$ is a scalar multiplier determining the amount of change in $\mathrm{X}$ for this iteration and is called the steplength. The step-length procedure is the minimization of a function with one variable. The golden section method [13] is an efficient method for the one-dimensional search. The rate of convergence is known and is very reliable for poorly conditioned problems.

Using FDM, for $\mathrm{S}$ (vector of search direction) to be usable, $\mathrm{F}(\mathrm{X})_{\mathrm{q}+1} \prec \mathrm{F}(\mathrm{X})_{\mathrm{q}}$ and clearly, such a search direction makes an angle greater than $90^{\circ}$ with the gradient vector of the objective function. Mathematically, the usability requirement becomes

$$
\nabla \mathrm{F}(\mathrm{X}) \cdot \mathrm{S} \leq 0 .
$$

and $\mathrm{S}$ is feasible if, for some move in that direction, the active constraints will not be violated. It means that the vector $\mathrm{S}$ must make an obtuse angle with all the constraints normal, so that

$$
\nabla g_{j}(X) \cdot S \leq 0
$$

The greatest reduction in $\mathrm{F}(\mathrm{X})$ can be achieved by finding $\mathrm{S}$ which minimizes the quantity in equation (37) while equation (38) meets with precise equality. By assuming the constraint is nonlinear and convex, a small move in this direction would violate the 
constraint. With these objectives, the following direction-finding problem is posed $[14,15]$.

Maximize $\quad \beta$

Subject to $\quad S^{T} \nabla g_{j}(X)+\theta_{j} \beta \leq 0 . \quad j \in J$

$$
\begin{aligned}
& \mathrm{S}^{\mathrm{T}} \nabla \mathrm{F}+\beta \leq 0 . \\
& -1 \leq \mathrm{S} \leq 1
\end{aligned}
$$

The scalars, $\theta_{\mathrm{j}}$, are referred to as 'push-off' factors which effectively push the design away from the active constraints and are defined as

$$
\theta_{\mathrm{j}}=\left[1-\frac{\mathrm{g}_{\mathrm{j}}(\mathrm{X})}{\varepsilon}\right]^{2} \theta_{0}
$$

where $\varepsilon$ is a small number. Usually $\theta_{0}=1$ is adequate for the nominal value of $\theta \mathrm{j}$.

The preceding formulation is suitable for the case that the design is inside the feasible domain. For the case that the point is infeasible, it needs a simple modification. It means the search direction will simultaneously minimize the objective function while overcoming the constraint violations. These considerations lead to the following statement of the direction-finding problem

$$
\begin{array}{ll}
\text { Maximize } & -\nabla F(X) . S+\Phi \beta \\
\text { Subject to } & \nabla g_{j}(X) . S+\theta_{j} \beta \leq 0 . \quad j \in J \\
& S^{\mathrm{T}} . S \leq 1
\end{array}
$$

Now the set $\mathrm{J}$ contains all violated constraints. The scalar $\Phi$ is weighting factor, determining the relative importance of the objective and the constraints. The choice of convergence criterion depends on the nature of the design problem and the required precision. For more details on FDM refer to Ref.[13]. 
Scaling and normalization also have significant influence on the performance of the optimisation method. To normalize constraints so that the order of each of them is unity improves the conditioning of the optimisation, as does scaling each constraint so that the gradient of the constraint is of the same order of magnitude as the gradient of the objective function. Scaling the design variables causes each variable to be of similar weight during optimisation. Normalization of the search direction provides a simple means of identifying the important parameters in the design. Therefore, scaling makes the optimisation more reliable.

\section{NUMERICAL RESULTS}

To apply the boundary element method for the minimization of elastic compliance of twodimensional anisotropic structures while satisfying some constraints upon weight, stresses and geometry, a general-purpose computer program has been developed. This is an advanced version of a shape optimisation program which was already produced by the author [6] for the weight minimization of anisotropic structures subject to stresses and geometrical constraints. This program uses an iterative technique and involves three major steps within each iteration: 1) an analysis of the stresses, displacements and elastic compliance of the structure for a given design 2) sensitivity analysis of the elastic compliance, stresses and weight, corresponding to possible changes in design 3) improvements to the design and regeneration of the boundary element mesh with isoparametric quadratic boundary elements. 
Five examples are selected to illustrate the use of the program. Four different materials $[16,17]$ are used to investigate the effect of engineering constants on the optimum shape design of the components. Every component is being treated as a lamina that has four engineering constants $E_{1}, E_{2}, G_{12}$ and $v_{12}$ with a lamina orientation angle of zero. See Table 2. Material 1 is isotropic but it is assumed as if it is anisotropic, $E_{1}=E_{2}$, $\mathrm{G}_{12}=\mathrm{E}_{1} / 2\left(1+v_{12}\right)$. The properties of materials 2,3 and 4 are as follows: for material 2, $\mathrm{E}_{1} / \mathrm{E}_{2}=1.0, \mathrm{G}_{12} / \mathrm{E}_{2}=2.94$ and $v_{12}=0.845$; for material 3, $\mathrm{E}_{1} / \mathrm{E}_{2}=40, \mathrm{G}_{12} / \mathrm{E}_{2}=0.492$ and $v_{12}=0.25$, for material $4 \mathrm{E}_{1} / \mathrm{E}_{2}=13.36, \mathrm{G}_{12} / \mathrm{E}_{2}=0.58$ and $v_{12}=0.295$. For some of the selected components, the optimum shape for the minimum compliance subject to weight and maximum stress constraint is compared to the optimum shape for the minimum weight subject to maximum stress constraint.

\subsection{Cantilever beam under distributed lateral load}

Fig. 2 shows a cantilever beam subject to a distributed lateral load. The objective is to find the optimum shape of edge $A B$, with $C D$ unchanged. The coordinates of points $\mathrm{P}_{1}$ to $\mathrm{P}_{7}$ which are equally spaced along $\mathrm{AB}$, are selected as the design variables. In reference [6], the weight of this beam was minimized subject to stress constraints. For verification purposes, this example was used to solve isotropic behaviour as a special case of anisotropy. The optimum shape produced from that study is presented in Fig. 3. The analysis was completed in ten iterations with a total weight reduction of $44 \%$. The maximum stress of the optimum shape was almost the same as the maximum stress of the initial design. 
In the present study, the elastic compliance of the cantilever beam is minimized, while the weight and maximum stress should not exceed more than 1.1 times the corresponding values of the initial design. Fig. 3 also shows the optimum shape of the beam when the elastic compliance is minimized. The analysis was carried out in 6 iterations with $50 \%$ reduction of the elastic compliance. The weight of the optimized shape nearly remained the same and its maximum stress was about $46 \%$ of the maximum stress of the initial design.

The preceding analysis was also performed for materials 2 and 3. Fig. 4 shows the optimum shape of the beam for each selected material. The optimum shape of the beam for material 1 is also presented in Fig. 4. For material 2, the elastic compliance has decreased by $51 \%$, the weight has increased by $13 \%$ and the maximum stress of the optimised shape is about $55 \%$ of the maximum stress of the initial design. For material 3 , the elastic compliance has decreased by $34 \%$, the weight has roughly remained the same and the maximum stress is $69 \%$ of the maximum stress of the initial design. It can be seen that material 3 , with the highest value of $E_{1} / E_{2}$, has the smallest reduction of elastic compliance during the optimisation process. The variation in compliance, as a proportion of the initial compliance during convergence to the optimum for each selected material, is shown in Fig. 5.

\subsection{Cantilever beam under load near free end}

Fig. 6a shows a cantilever beam with a uniformly distributed load at a small portion of its upper side near the free end. The objective is to minimize the elastic compliance of the beam while the maximum stress and the weight of the beam are not to exceed 1.3 times 
the corresponding values of the original design. Edge $A B$ is to altered. Materials 1 to 3 are selected for this analysis. Figs. 7 and 8 show the optimised shapes of the beam and compliance iteration history, respectively. The analysis was carried out in 9, 13 and 10 iterations with 61,76 and $40 \%$ reduction of elastic compliance for materials 1, 2 and 3, respectively. The weight of the beam is increased by 25,34 and $30 \%$ for materials 1,2 and 3, respectively. However, the maximum stress has decreased by $59 \%$ for material 1 , $69 \%$ for material 2 , and $40 \%$ for material 3 . It can be observed that in this case the minimization of the elastic compliance has been at the expense of the weight increase, whereas the maximum stress in each case has greatly reduced.

\subsection{Circular plate with a cavity subject to internal pressure}

Fig. 9 shows a circular plate with a central cavity subject to internal pressure. The objective is to minimize the elastic compliance of the plate with constraints upon stresses and weight. Materials 2 and 3 from Table 2 are chosen for the analysis. In each case the optimization procedure is carried out with the maximum allowable limit on each constraint remaining below 1.2 times the corresponding values of the original design. The geometry is symmetric about both coordinate axes, so only a quarter of the plate needs to be modelled. See Fig. $9 \mathrm{~b}$. The radii of points $\mathrm{P}_{1}$ to $\mathrm{P}_{5}$ located on the inner boundary at the fixed angles, as shown in Fig. 9c, are selected as the design variables. Fig. 10 shows the initial geometry and optimum shapes of the cavity in the plate for the selected materials. Fig. 11 shows the elastic compliance $\left(\mathrm{E}_{\mathrm{S}} / \mathrm{E}_{\mathrm{s} 0}\right)$ iteration history for the plate. For material 2, the analysis was carried out in 11 iterations with $19 \%$ reduction of the elastic compliance and for material 3, the optimum shape was obtained in 14 iterations with $23 \%$ reduction of the elastic compliance. For each material the maximum 
stress of the optimized geometry nearly remained unaffected, whereas there was about $1 \%$ increase in the weight of the plate after optimization.

In Ref. [6], the minimum weight of the plate subject to maximum stress constraint was obtained. Material 2 was selected for the analysis. Here, the minimum weight of the plate for material 3 is also obtained. These results are also presented in Fig. 10. The reduction in the weights, for materials 2 and 3 , were $16 \%$ and $18 \%$, respectively, while the maximum stresses almost remained unaffected.

Although the geometry and boundary conditions of the circular plate with a cavity are symmetric, the results for the optimum shape design are not symmetric (see Fig. 10). This is the consequence of the anisotropic behaviour of the material.

\subsection{Connecting rod}

Fig. 12a shows a connecting rod subject to a uniform internal pressure of 1 unit, over the semi-circular region, assumed to be in contact with the pin, and a uniform tension at the remote end of the rod. The objective is to design a connecting rod profile in the transition region between the pin hole and the loaded end of the rod. Due to the symmetry, only the upper half of the model is considered. The design variables are the coordinates of points $\mathrm{P}_{1}$ to $\mathrm{P}_{6}$ in Fig. $12 \mathrm{~b}$. Materials 1 to 3 were selected for the analysis.

First, the weight of the rod was minimized with the maximum stress not to exceed 1.1 times the maximum stress of the original geometry. Fig. 13a shows the optimum shape of the rod for each selected material, when the weight has been minimized. The weight 
reduction, for materials 1 to 3 , were 44, 53 and 26\%, respectively. Fig. 14 shows the weight $\left(\mathrm{W} / \mathrm{W}_{0}\right)$ iteration history of the plate for the selected materials. For all three cases the optimization procedure ended in 9 iterations.. The maximum stress for the final design was reduced by $70 \%$ and $67 \%$ for materials 1 and 2, respectively, and by $48 \%$ for material 3. The highest weight reduction was for material 2.

Second, the elastic compliance of the rod was minimized, while the weight and maximum stress of the plate were limited not to exceed 1.1 times the corresponding values of the original design. Fig. 13b shows the optimum shapes of the connecting rod for the selected materials, when the elastic compliance has been minimized. Fig. 15 shows the elastic compliance iteration history of the plate for the selected materials. It can be seen that the elastic compliance has been reduced by $92-93 \%$, regardless of the material properties. However, not only has the elastic compliance of the optimized shape reduced but also the weight and the maximum stress of the rod have been decreased. For materials 1 and 2, the weight of the final design is about $66 \%$ of its original value while for material 3 is $74 \%$ of the weight of the original design. It can also be observed that the maximum stress has decreased by 48,60 and $67 \%$ for materials 1 to 3 , respectively. For all three cases the optimization procedure ended in 14 iterations. From this case study, it can be concluded that the minimization of the elastic compliance is not always at the expense of the weight increase. However, the loaded edge of the connecting rod, which is subject to a distributed load, is decreased during the optimization procedure. 


\subsection{Link plate}

This example involves a link plate loaded through pin joints at its two ends. The plate experiences both direct tensile and bending moment loads. See Fig 16. Taking advantage of the symmetry, only the right half of the plate is analysed. The loading applied is a uniform internal pressure of one unit, over the semicircular region of each hole, assumed to be in contact with the pin. The coordinates of 13 points, P1 to P13, are selected as design variables.

In reference [6], the optimum shape of the outer boundary of the link plate was obtained to minimize its weight subject to stress constraints. Materials 2 and 4 from Table 1 were selected for the analysis. For each selected material, the optimization procedure was carried out with the maximum allowable limit on the maximum stress not to exceed 1.3 times the maximum stress of the original geometry. The analysis of the material 2 was completed in ten iterations, with $47 \%$ reduction in the weight. For material 4 , the analysis was completed in seven iterations with $24 \%$ weight reduction. For both cases, the maximum stresses of the optimised shapes were about $25-28 \%$ higher than the maximum stresses of the original geometries. The optimum shapes produced from that study are shown in Fig. 17.

Here, the compliance of the link plate is minimized while the maximum allowable limits on stress and weight are not to exceed 1.1 times the corresponding values of the original design. Fig. 18 shows the variation in compliance, as a proportion of the initial compliance during the optimisation procedure, which shows almost the same trend for the selected materials (2 and 4). The analysis of material 2 is completed in 9 iterations with $35 \%$ reduction of the elastic compliance, having 10 and $8 \%$ increase in the weight and stress, respectively. The analysis of material 4 is completed in 9 iterations with $40 \%$ 
reduction of the elastic compliance, having 2 and $10 \%$ increase in the weight and stress, respectively. It can be observed that during the minimization of the elastic compliance, regardless of the material properties, the weight and stress of the link plate have increased. Fig. 17 shows that the optimum shapes for materials 2 and 4 are almost identical. Therefore, for the link plate, the minimization of the compliance has been at the expense of increase in the weight and the maximum stress.

\section{SUMMARY AND CONCLUSION}

Following a brief review of the mathematical basis of the boundary integral equation method for two-dimensional elastic anisotropic materials, analytical differentiation of the boundary integral equation was carried out. Shape design sensitivity analysis was performed to compute the derivatives of displacements, stresses, weight and elastic compliance with respect to changes of design variables, which were the positions of some of the boundary nodes. To maximize the stiffness, the elastic compliance was minimized while satisfying some constraints upon weight, stresses and geometry. The results were compared with the results produced based on the weight minimization subject to stresses and geometrical constraints. Five examples have been analysed and the results are presented. Four different anisotropic materials were employed for the analysis. It is shown that the optimum shape of a composite structure, for maximum stiffness and minimum weight, not only depends on the loading conditions but also on the material properties. 


\section{REFERENCES}

1. A. Tafreshi, "Design optimisation using the boundary element method", PhD Thesis, Imperial College of Science, Technology and Medicine, University of London, 1990

2. A. Tafreshi and R.T. Fenner, "Design optimisation using the boundary element method", J. Strain Analysis, V26, N4, pp231-241, 1991

3. A. Tafreshi, and R.T. Fenner, "Design sensitivity analysis using the boundary element method”, J. Strain Analysis, V28, N4, pp 283-291, 1993

4. A. Tafreshi and R.T. Fenner, "General-purpose computer program for shape optimisation of engineering structures using the boundary element method", Computers and Structures, V56, No. 5, pp 713-720, 1995

5. A. Tafreshi, " Shape design sensitivity analysis of 2D anisotropic structures using the boundary element method", Engineering Analysis with Boundary Elements, V26, pp237-251, 2002

6. A. Tafreshi,"'Shape optimisation of two-dimensional anisotropic structures using the boundary element method", Journal of Strain Analysis, V38, 3, 219-232, 2003

7. R. F. Gibson, "Principles Of Composite Material Mechanics", McGraw-Hill, Inc., 1994

8 S.G. Lekhnitskii, “Theory of Elasticity Of An Anisotropic Elastic Body”, HoldenDay, San Francisco, California, Inc. 1963

9. M.D. Snyder and T.A. Cruse, "Boundary-integral equation analysis of cracked anisotropic plates”, Int. J. Fracture, V11, No. 2, pp315-328, 1975 
10. R.B. Wilson and T.A. Cruse, "Efficient implementation of anisotropic three dimensional boundary integral equation stress analysis", International Journal for Numerical Methods in Engineering, Vol.12, pp1383-1397, 1978

11. C.L. Tan, C.L. Gao and F.F. Afagh, "Anisotropic stress analysis of inclusion problems using the boundary integral equation method", Journal of Strain analysis, V27, No. 2, 67-76, 1992

12. T.A. Cruse, "Boundary Element Analysis in Computational Fracture Mechanics", Kluwer, Dordrecht, Academic Publishers, 1988

13. G.N. Vanderplaats, "Numerical Optimisation Techniques for Engineering Design with Applications, McGraw-Hill, London 1984

14. G.Zoutendijk, "Methods of feasible directions", Elsevier, Amsterdam,1960

15. G.N.Vanderplaats and F.Moses, "Structural optimisation by methods of feasible directions", Journal of Computers and Structures, V13, pp739-755, July 1973

16. Tafreshi, A., "Efficient modelling of delamination buckling in composite cylindrical shells subject to axial compression”, Composite Structures, 64/3-4, 511-520, 2004

17. Tafreshi, A., "Delamination buckling and post-buckling analysis of composite cylindrical shells subject to external pressure", Journal of thin-walled structures, In Press 


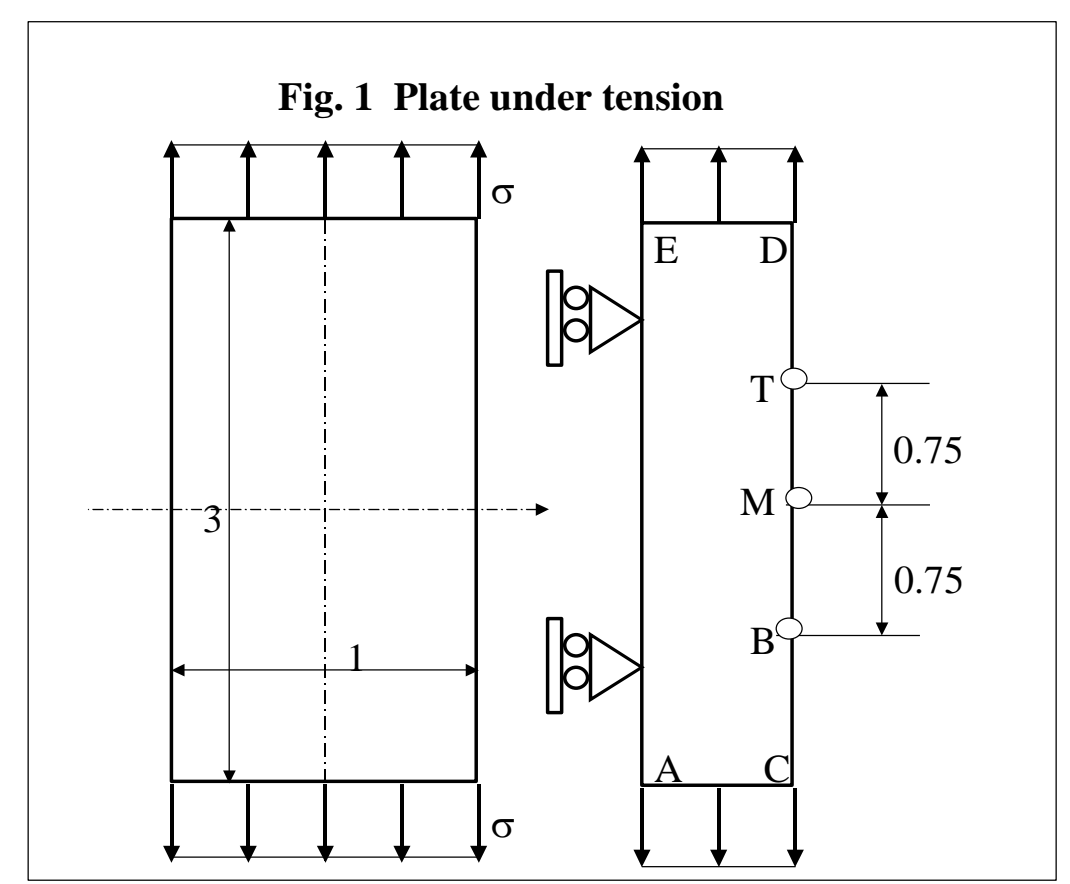

Fig. 2 Cantilever beam under lateral load

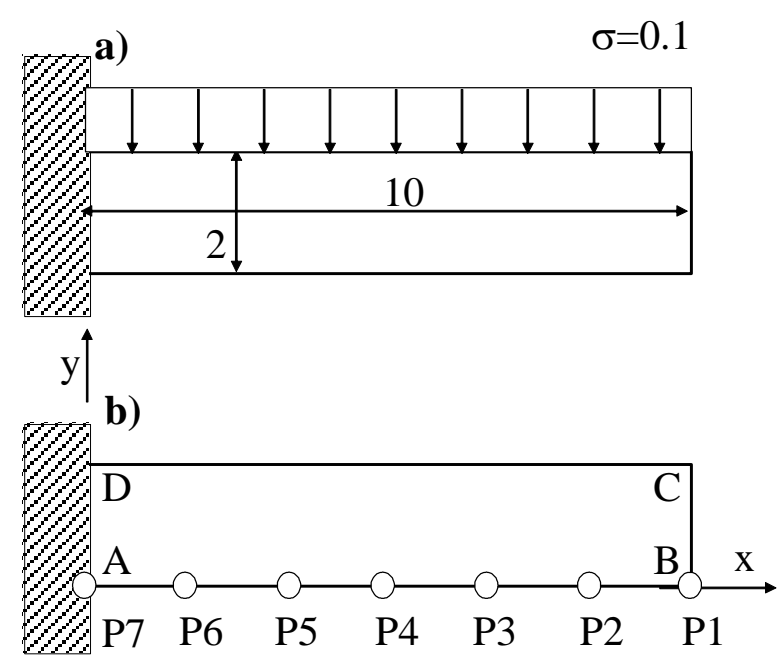


Fig. 3 Optimum shape design of an isotropic cantilever beam under distributed lateral load

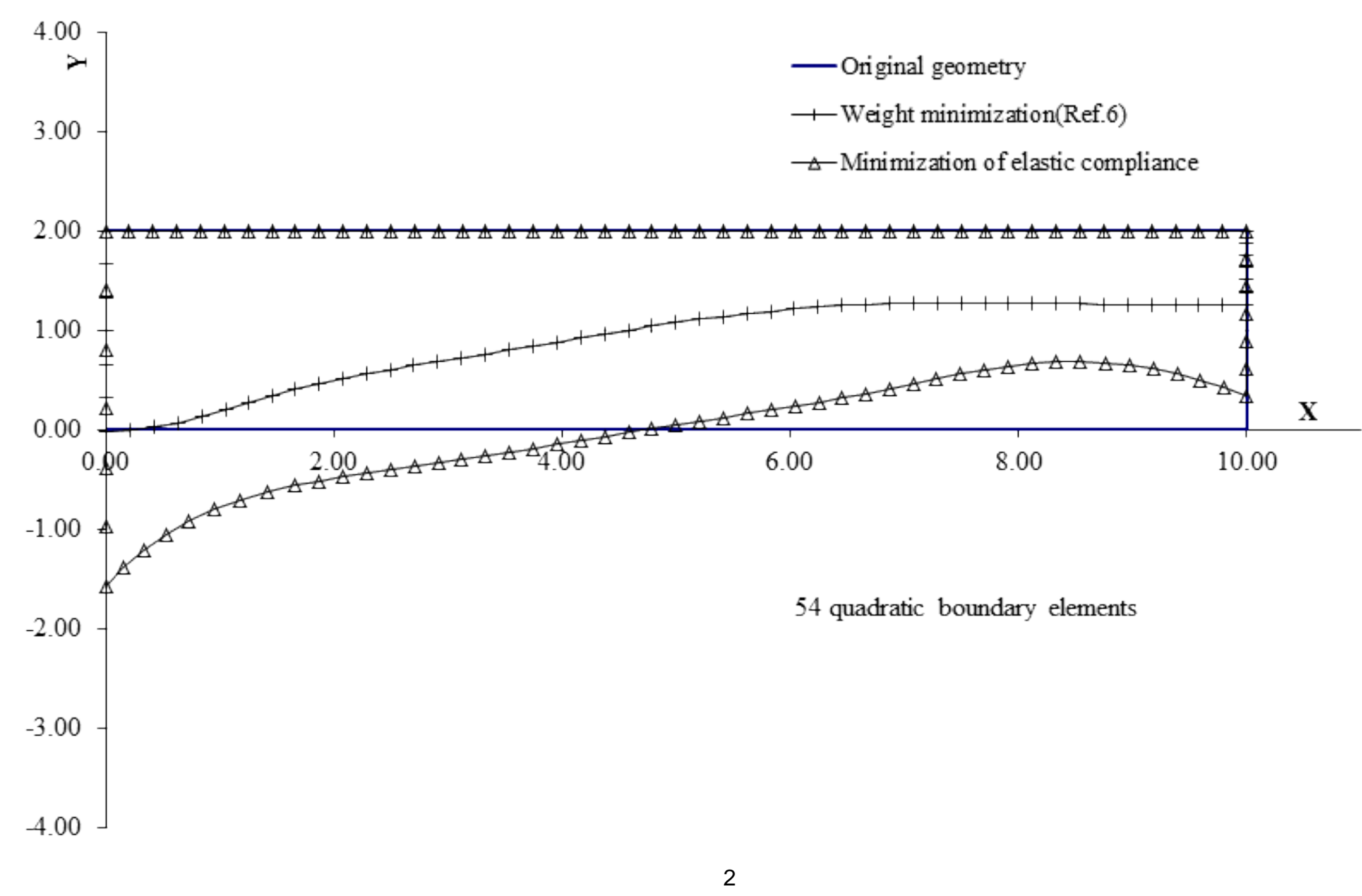


Fig. 4 Optimum shape design of a cantilever beam under distributed lateral load, minimization of elastic compliance

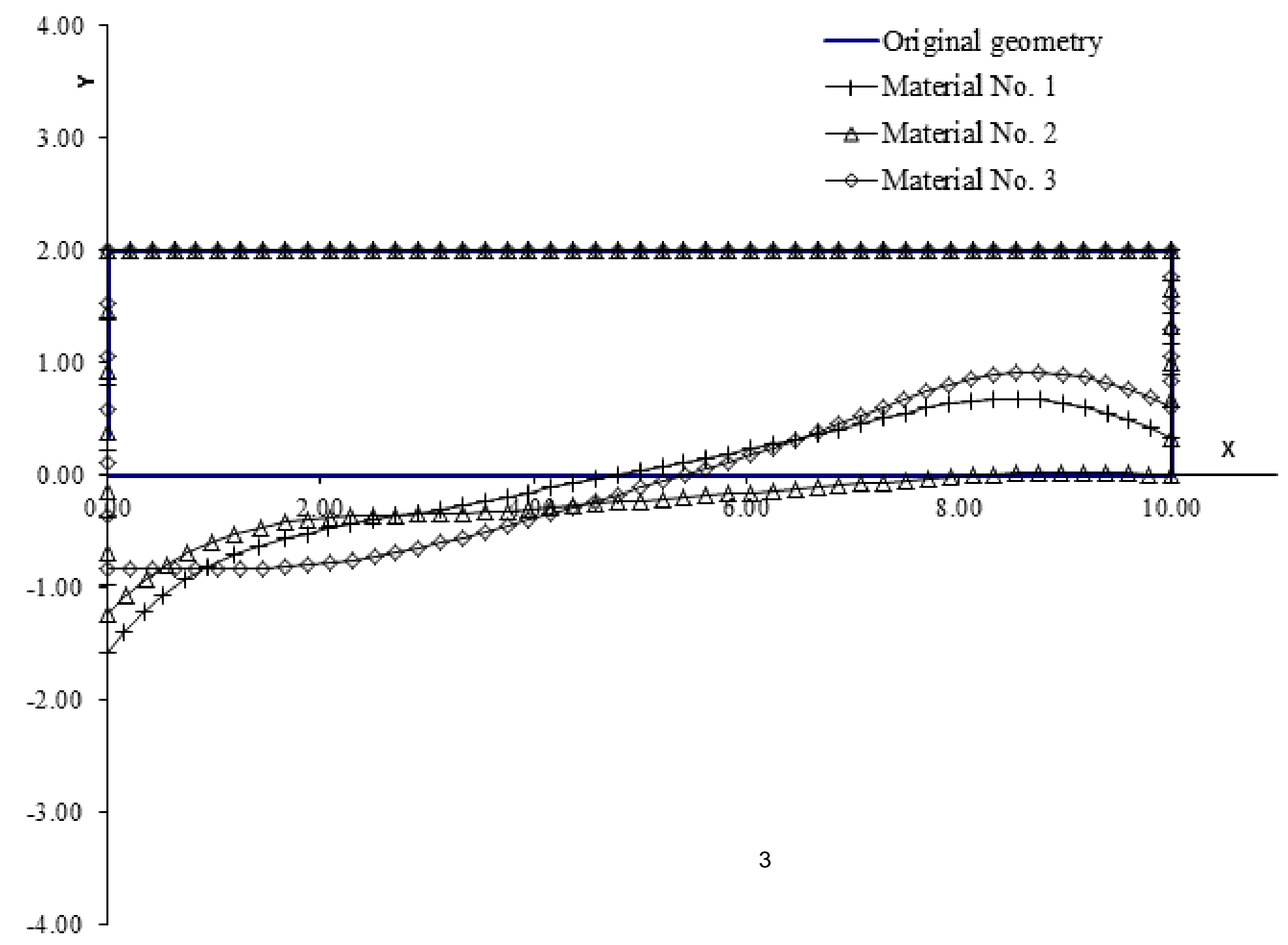


Fig. 5 Compliance iteration history for the cantilever beam under distributed lateral load

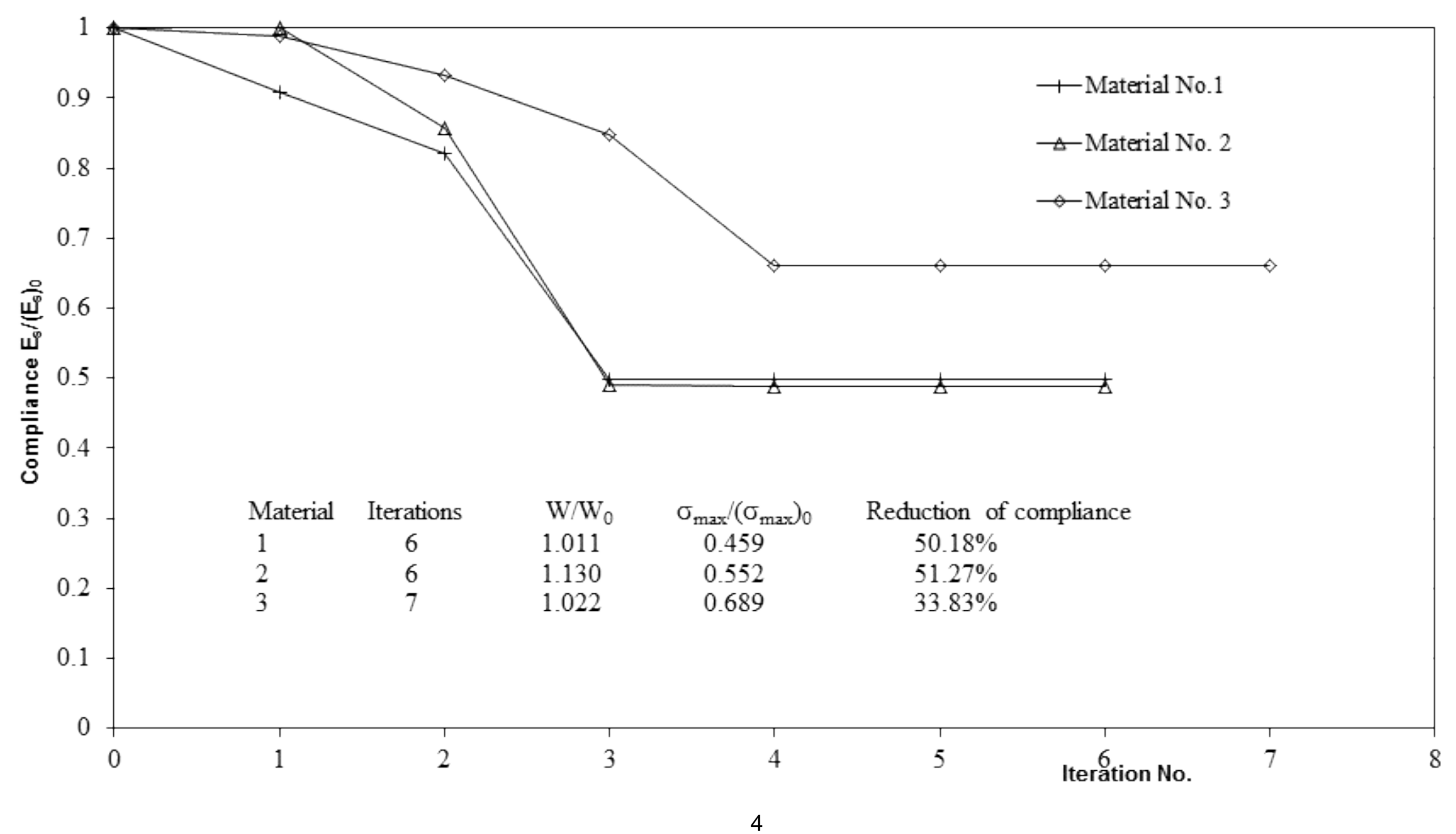


Fig. 6 Cantilever beam under load near free end

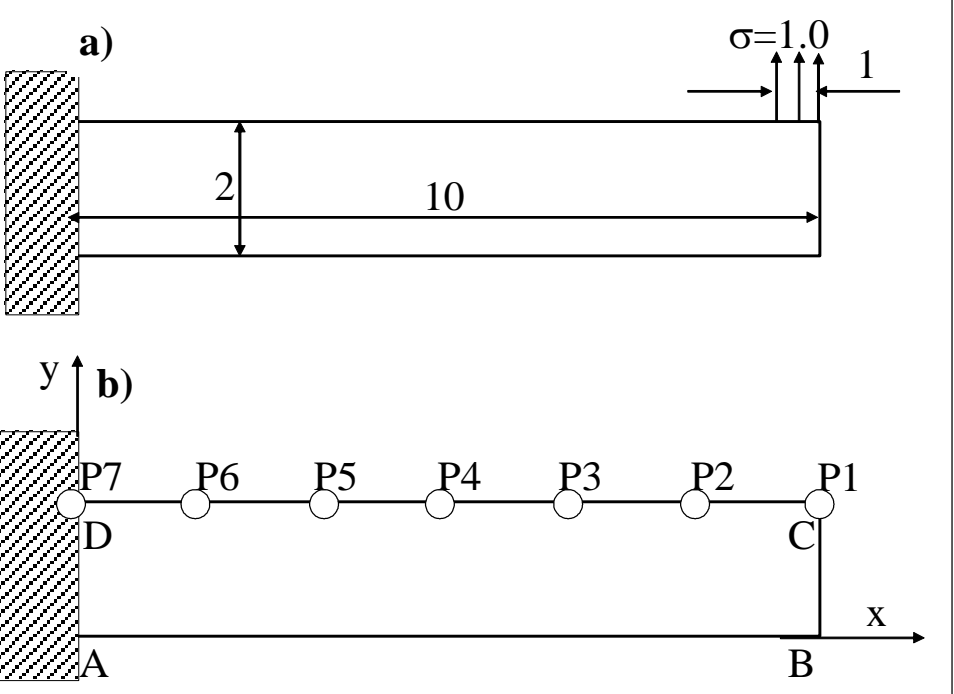


Fig. 7 Optimum shape design of a cantilever beam under load near free end, minimization of elastic compliance

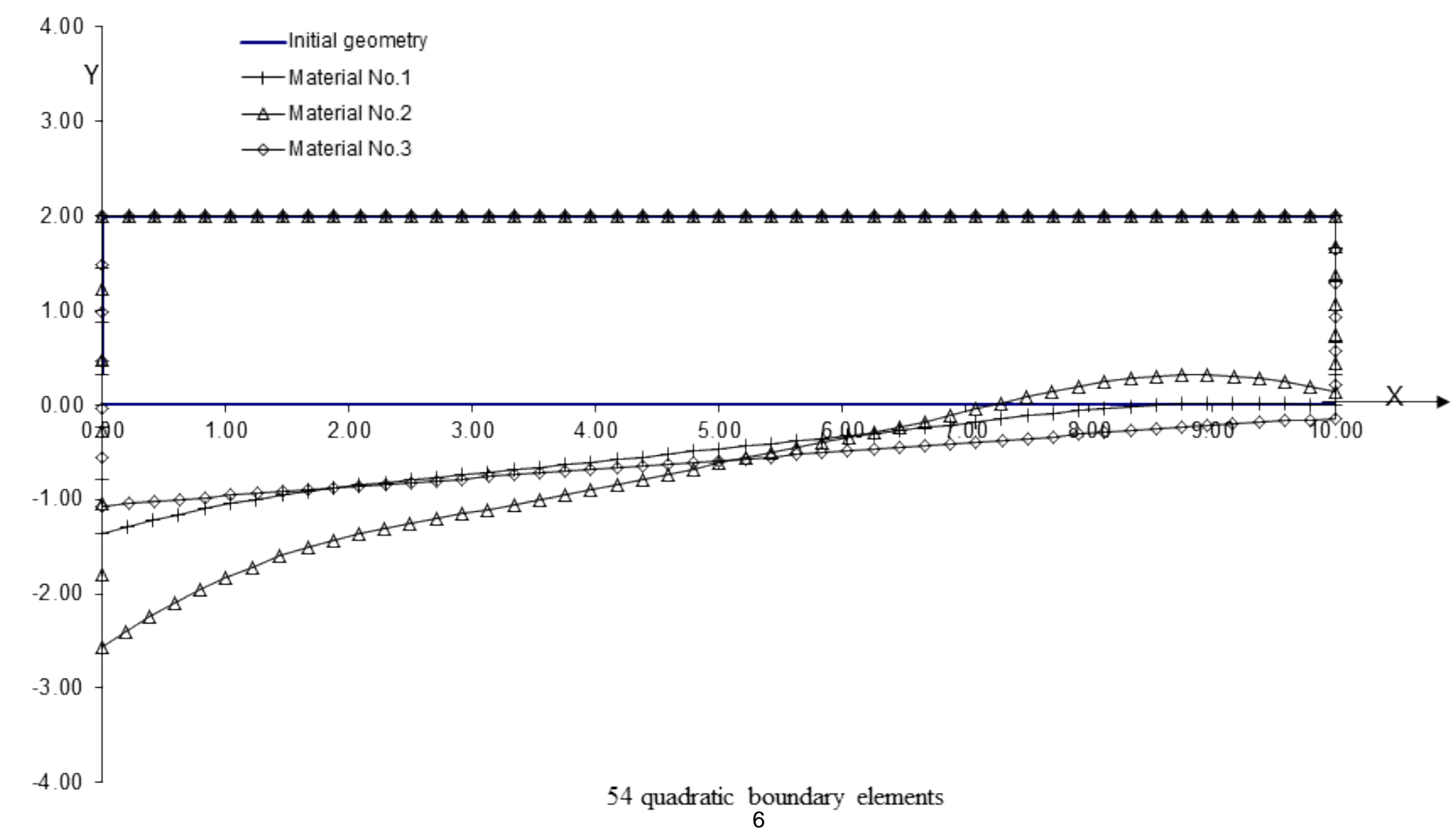


Fig. 8 Compliance iteration history for the cantilever beam under load near free end

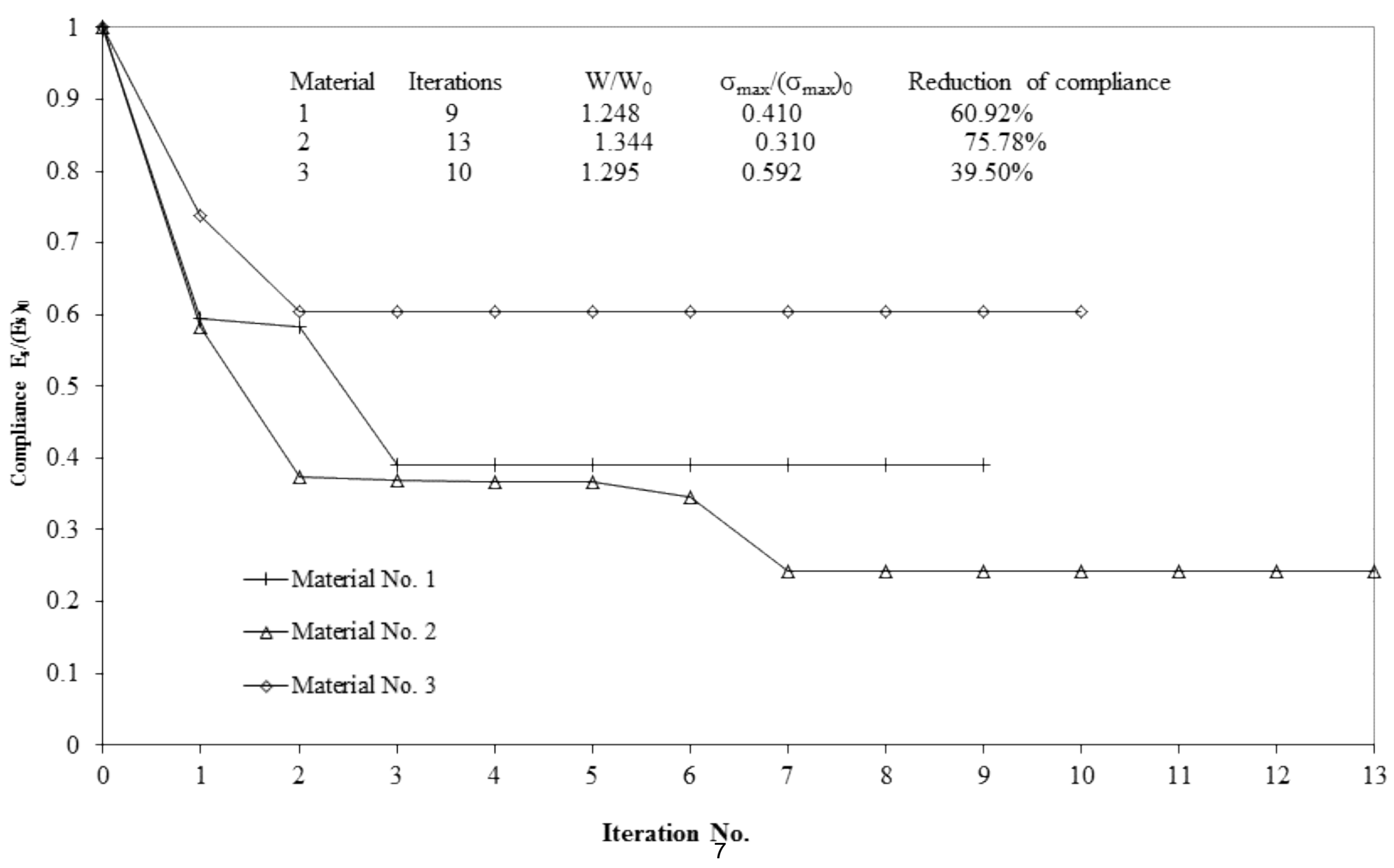




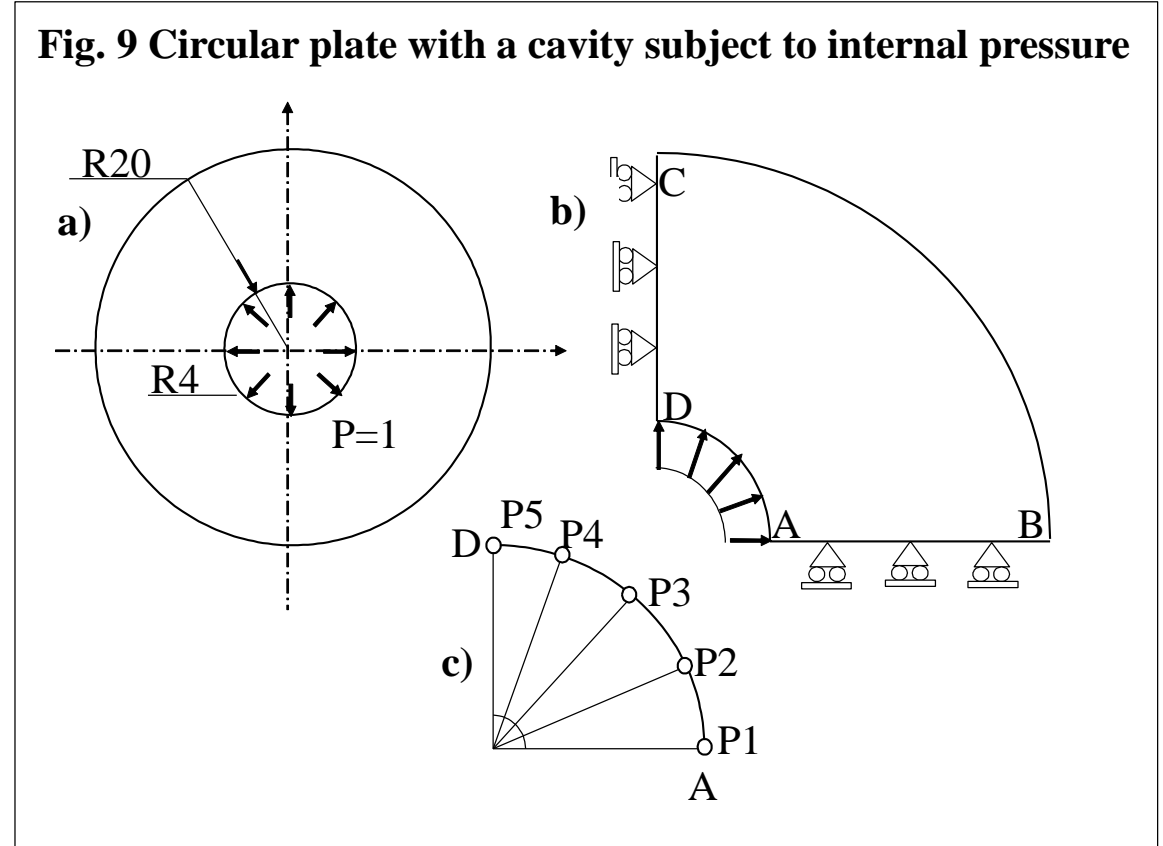




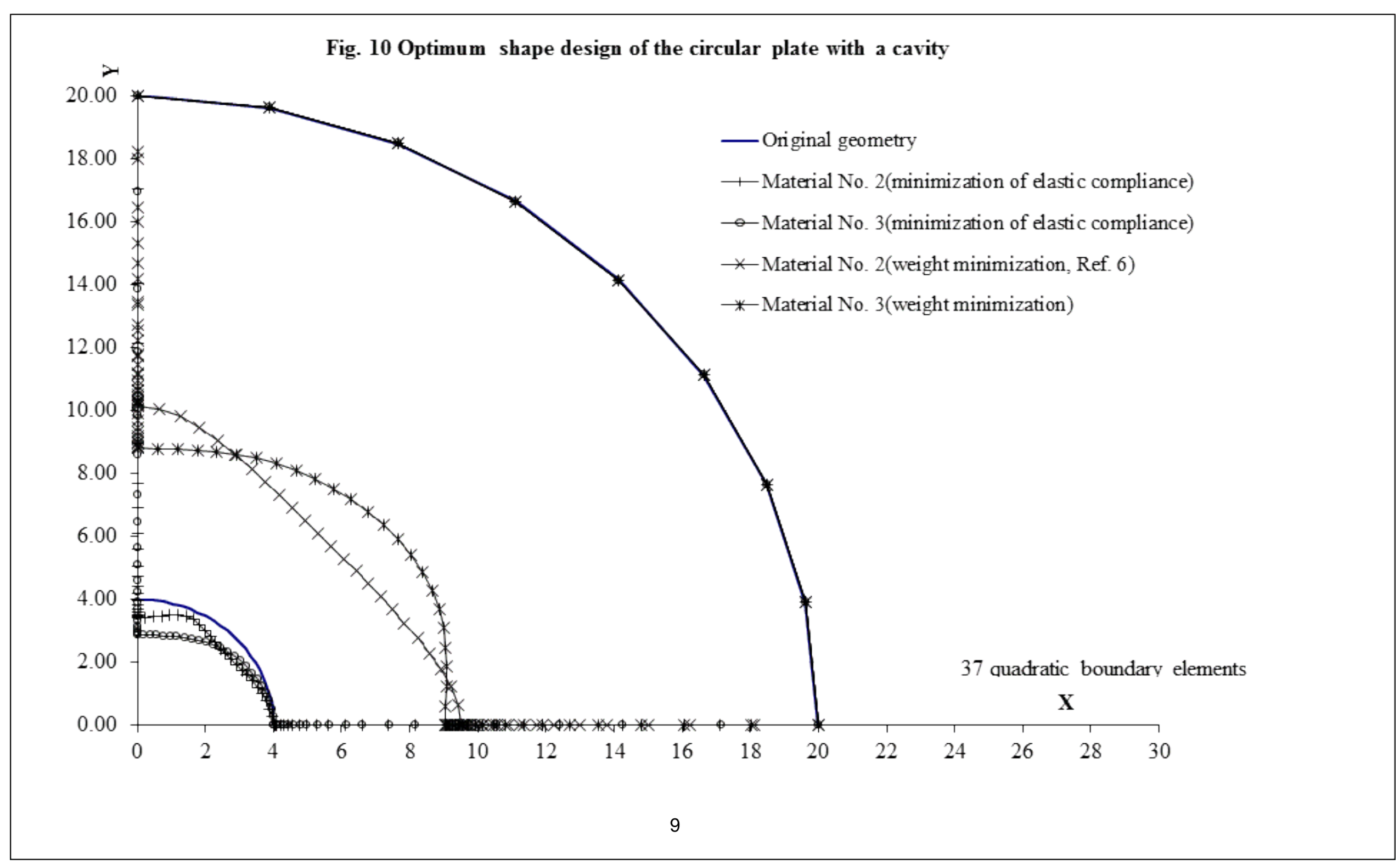


Fig. 11 Elastic compliance iteration history for the circular plate with a cavity

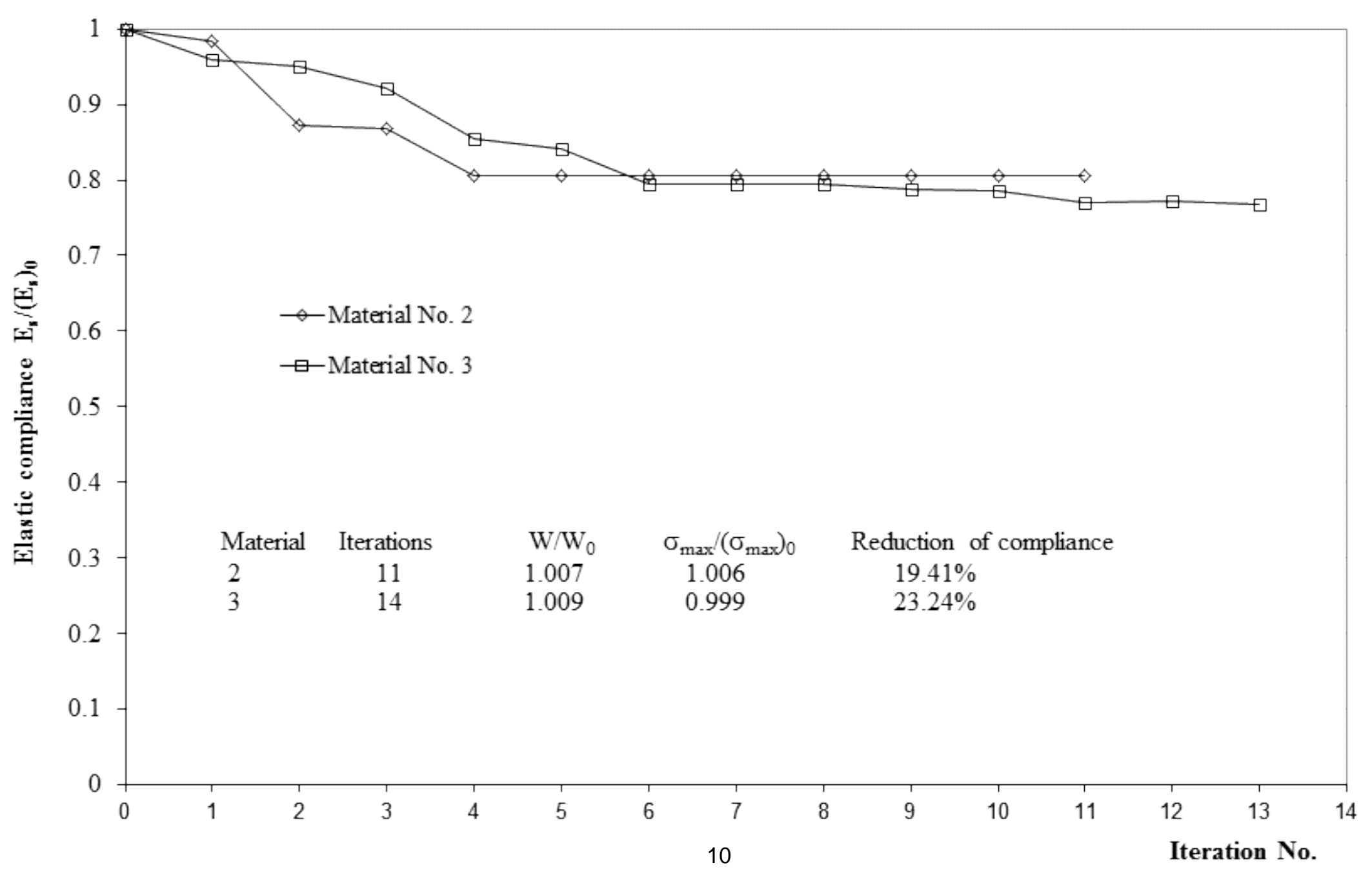




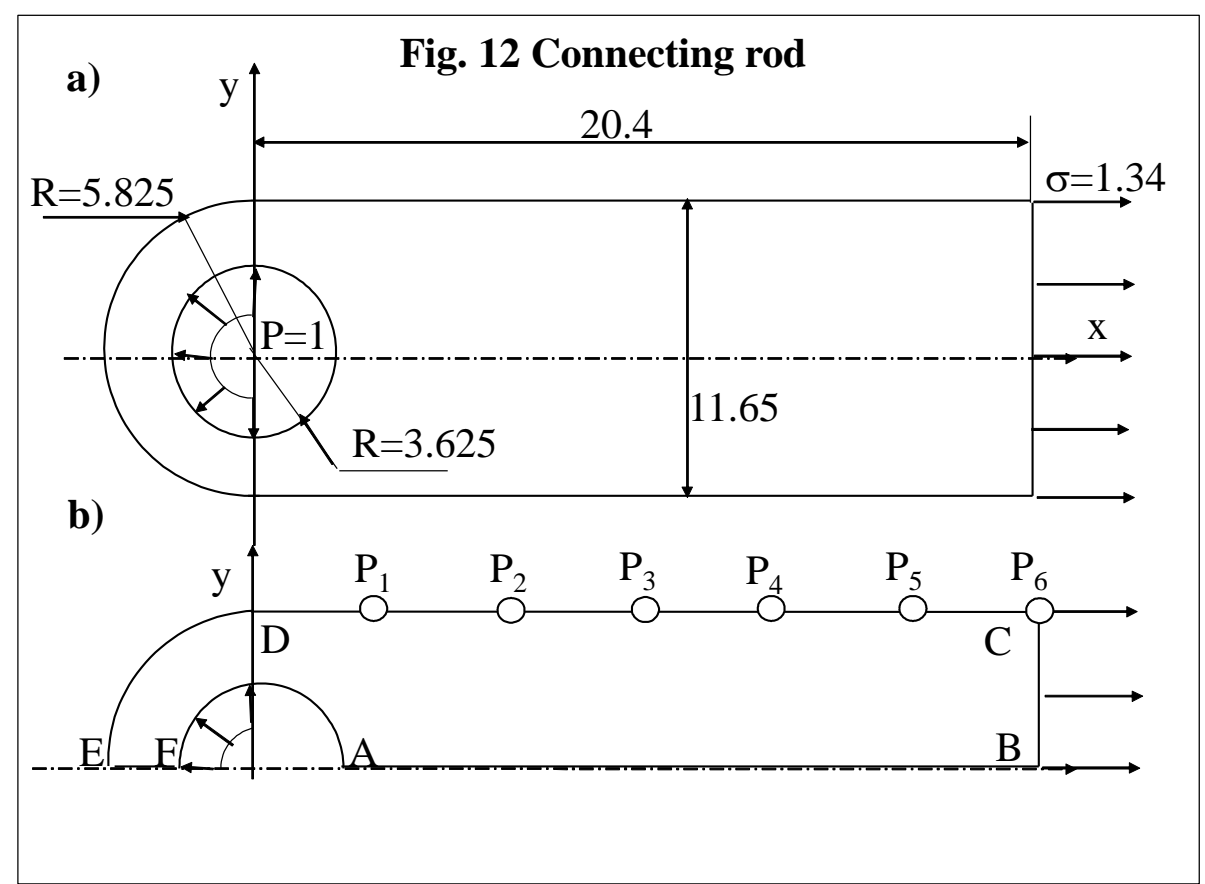


Fig. 13a Optimum shape design of the connecting rod, weight minimization

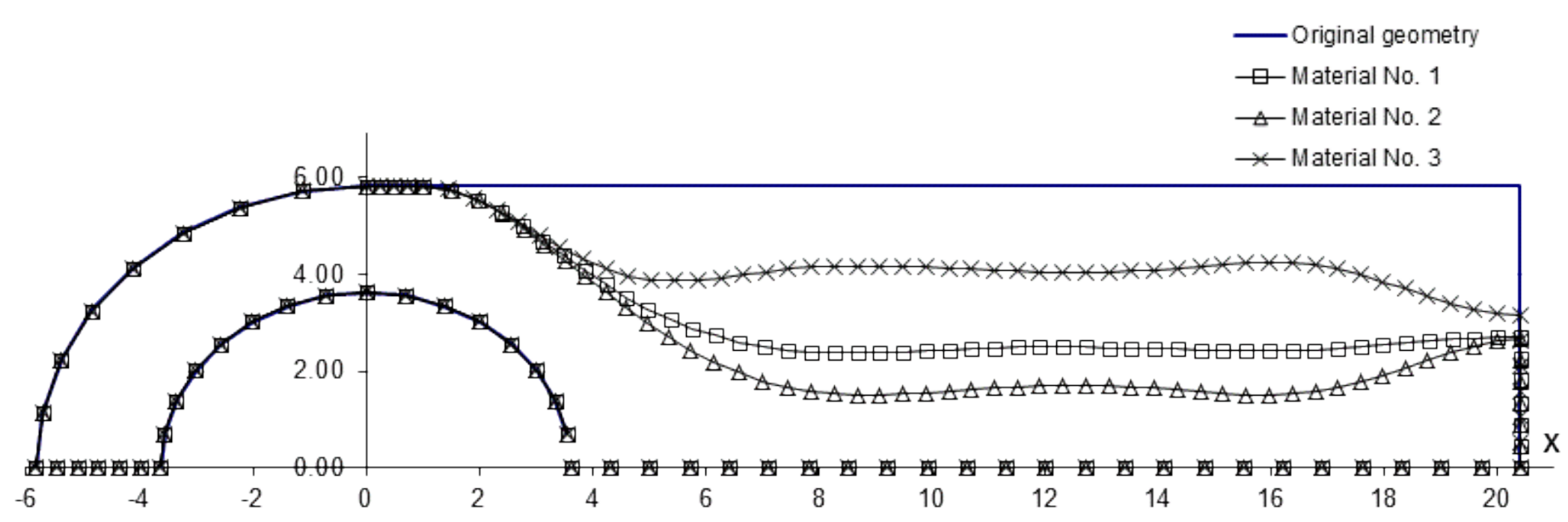

56 quadratic boundary elements 
Fig. 13b Optimum shape design of the connecting rod, minimization of elastic compliance

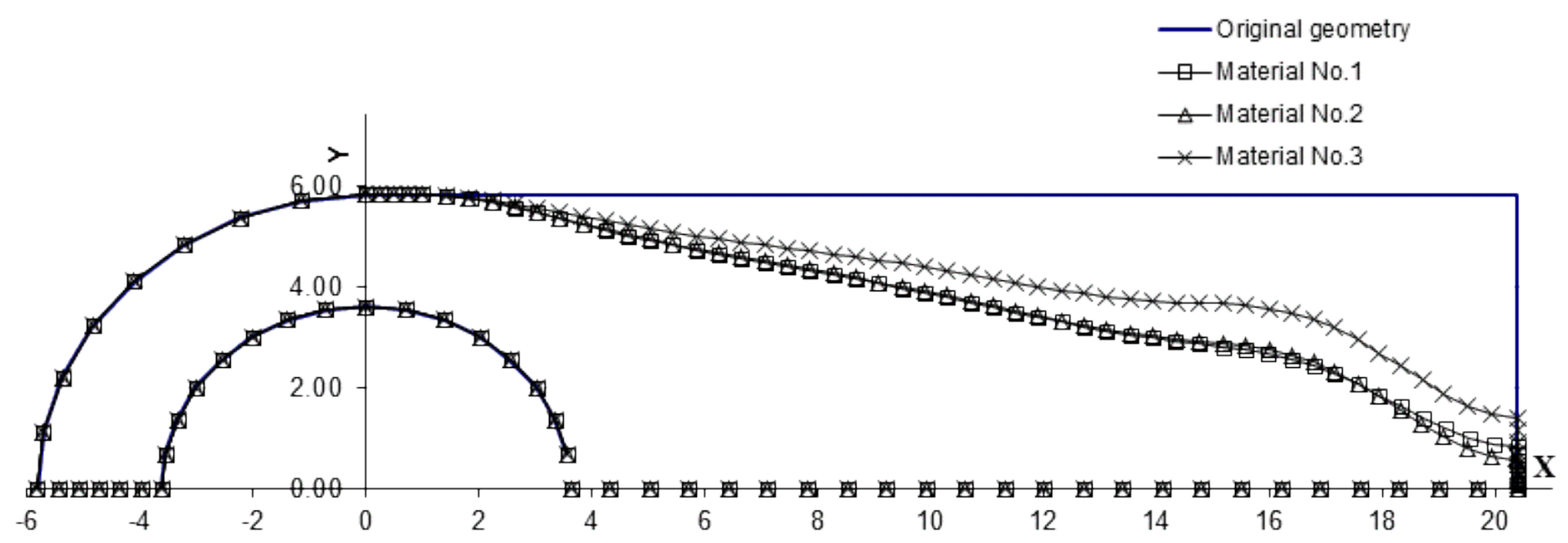

56 quadratic boundary elements 
Fig. 14 Weight iteration history of the connecting rod

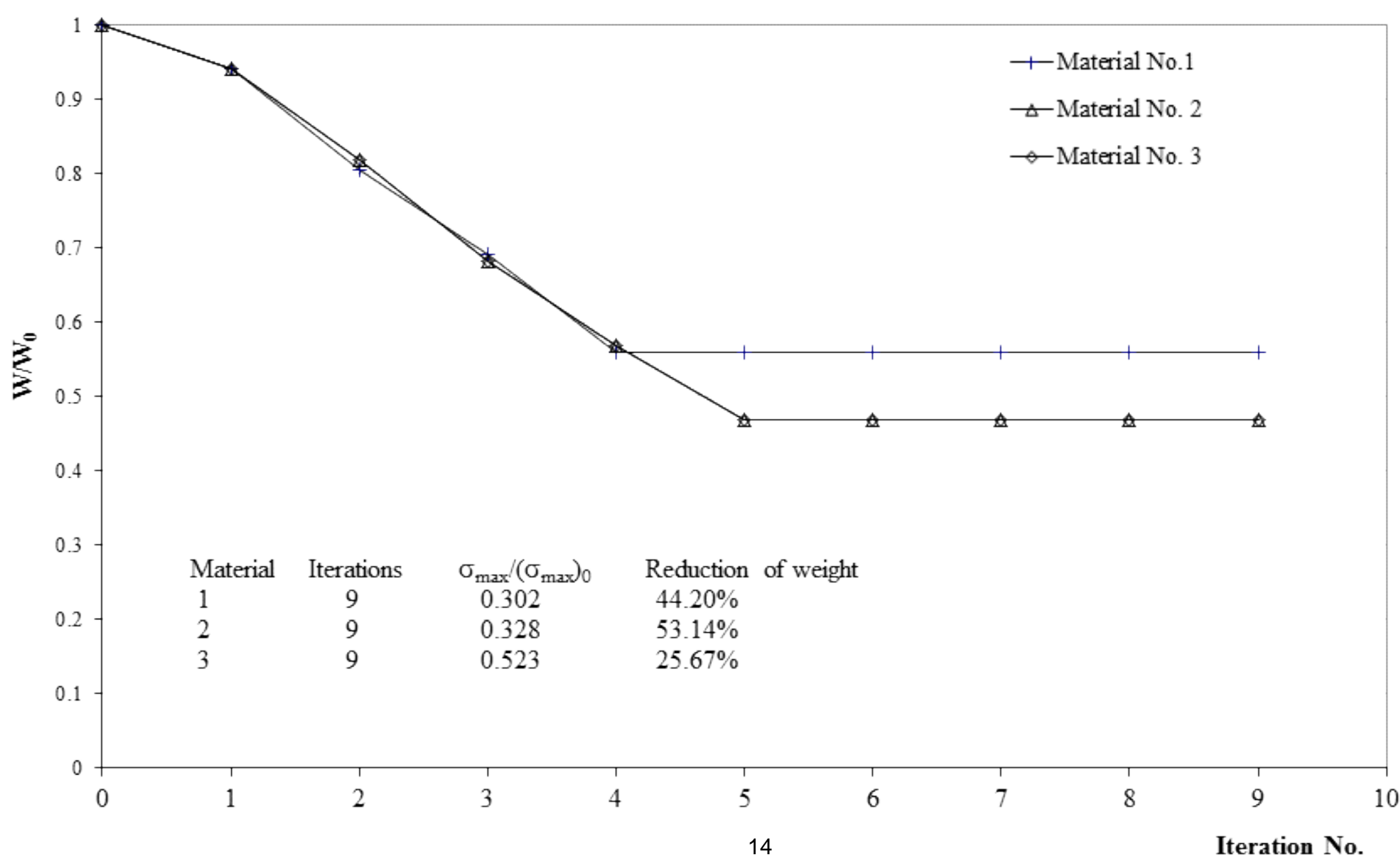


Fig. 15 Elastic compliance iteration history for the connecting rod

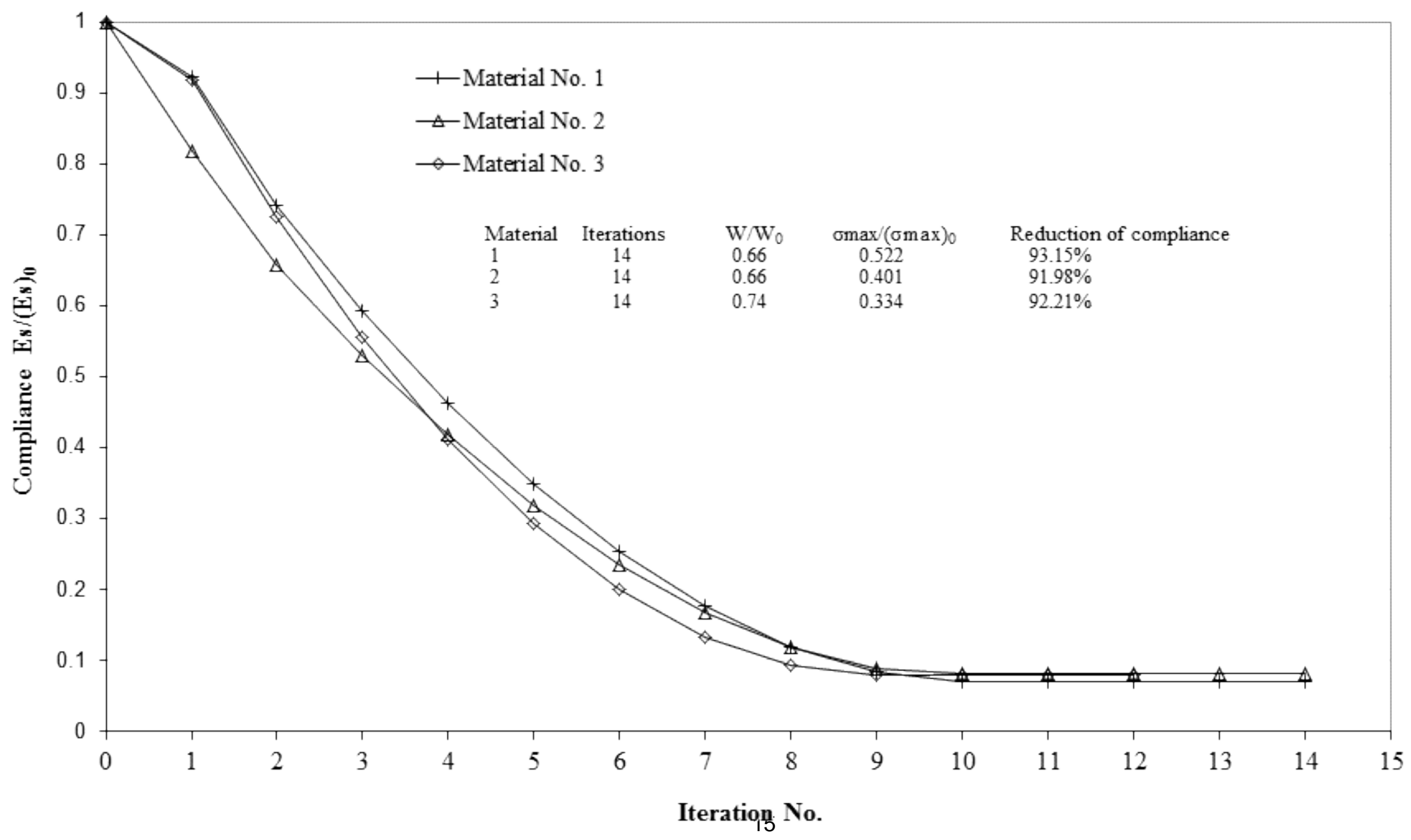




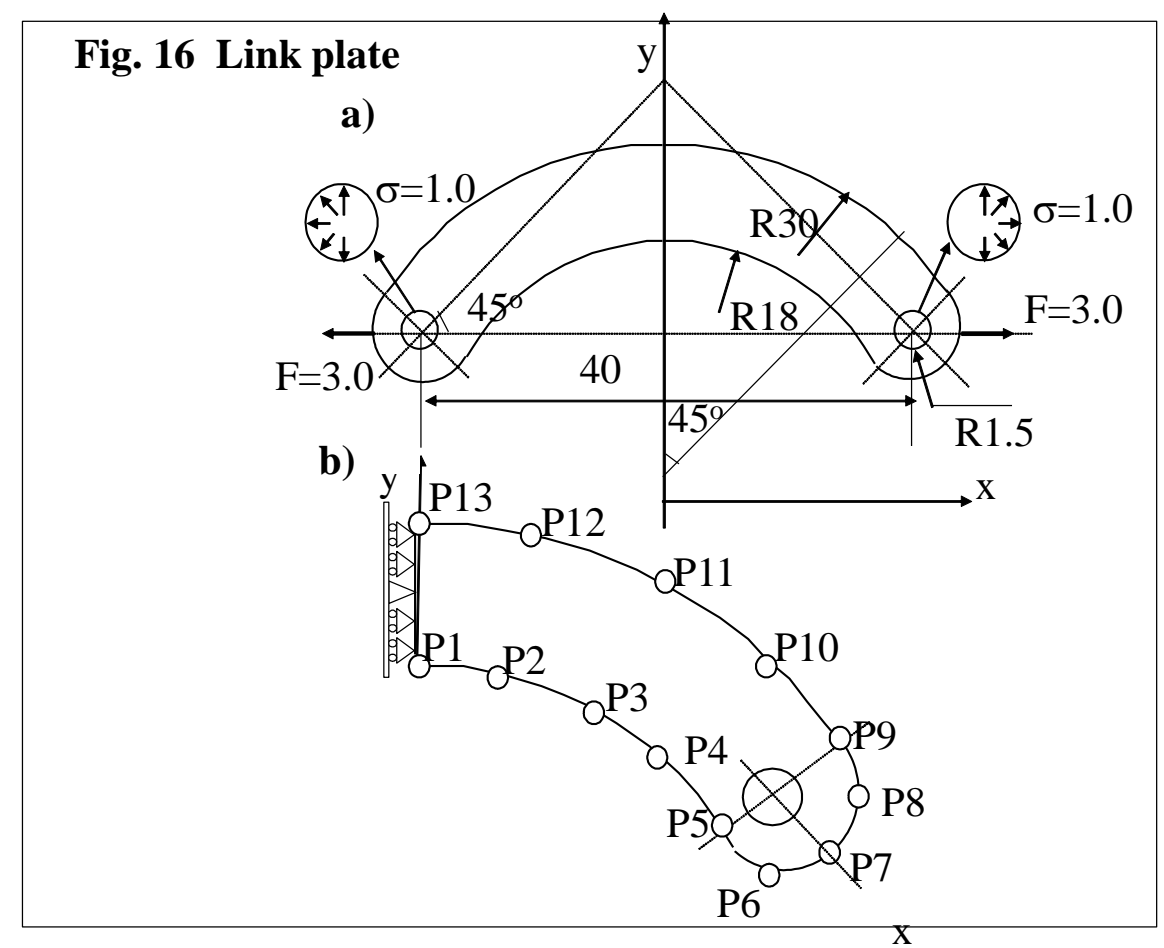


Fig. 17 Optimum shape design of the link plate

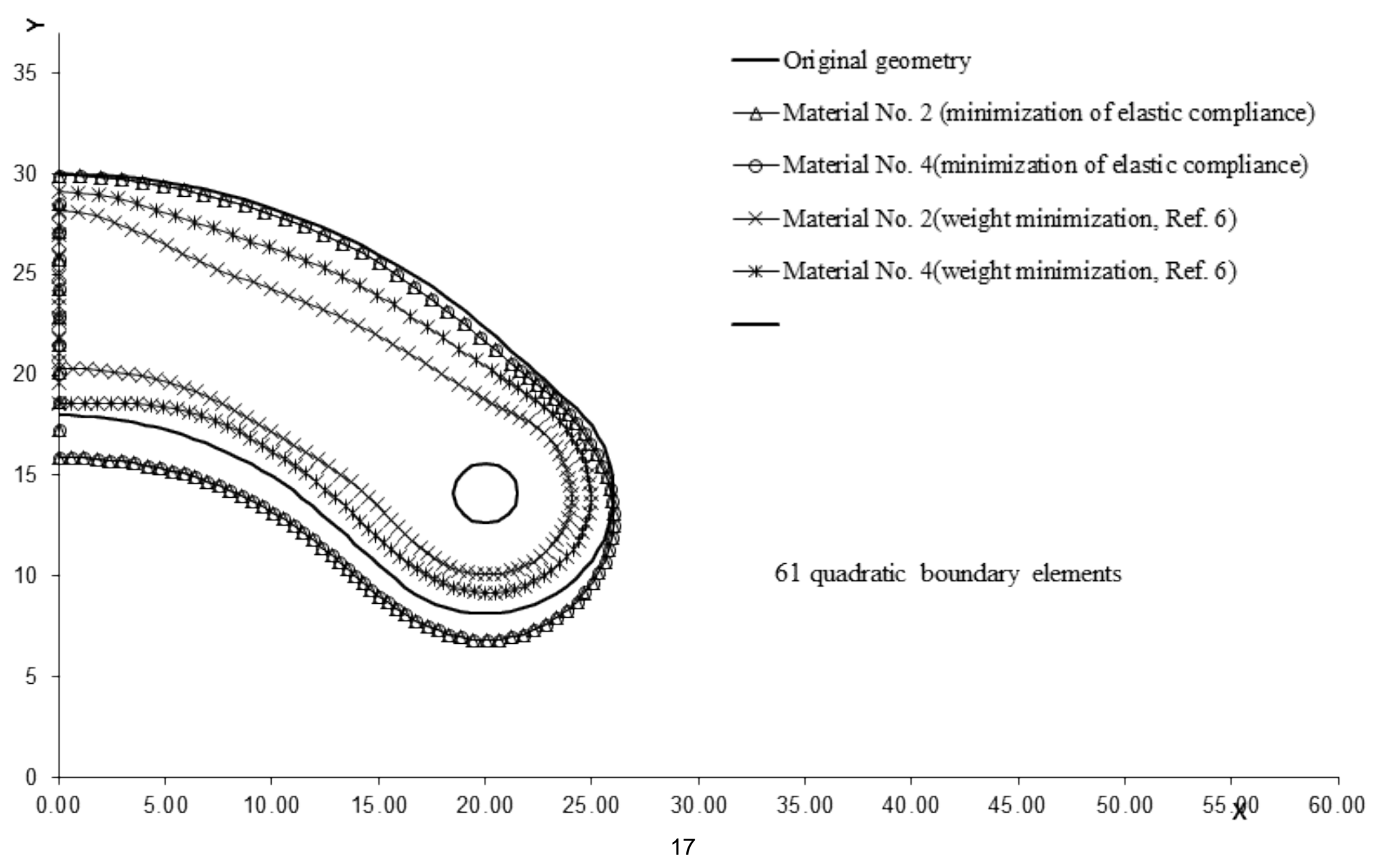


Fig. 18 Compliance iteration history for the link plate

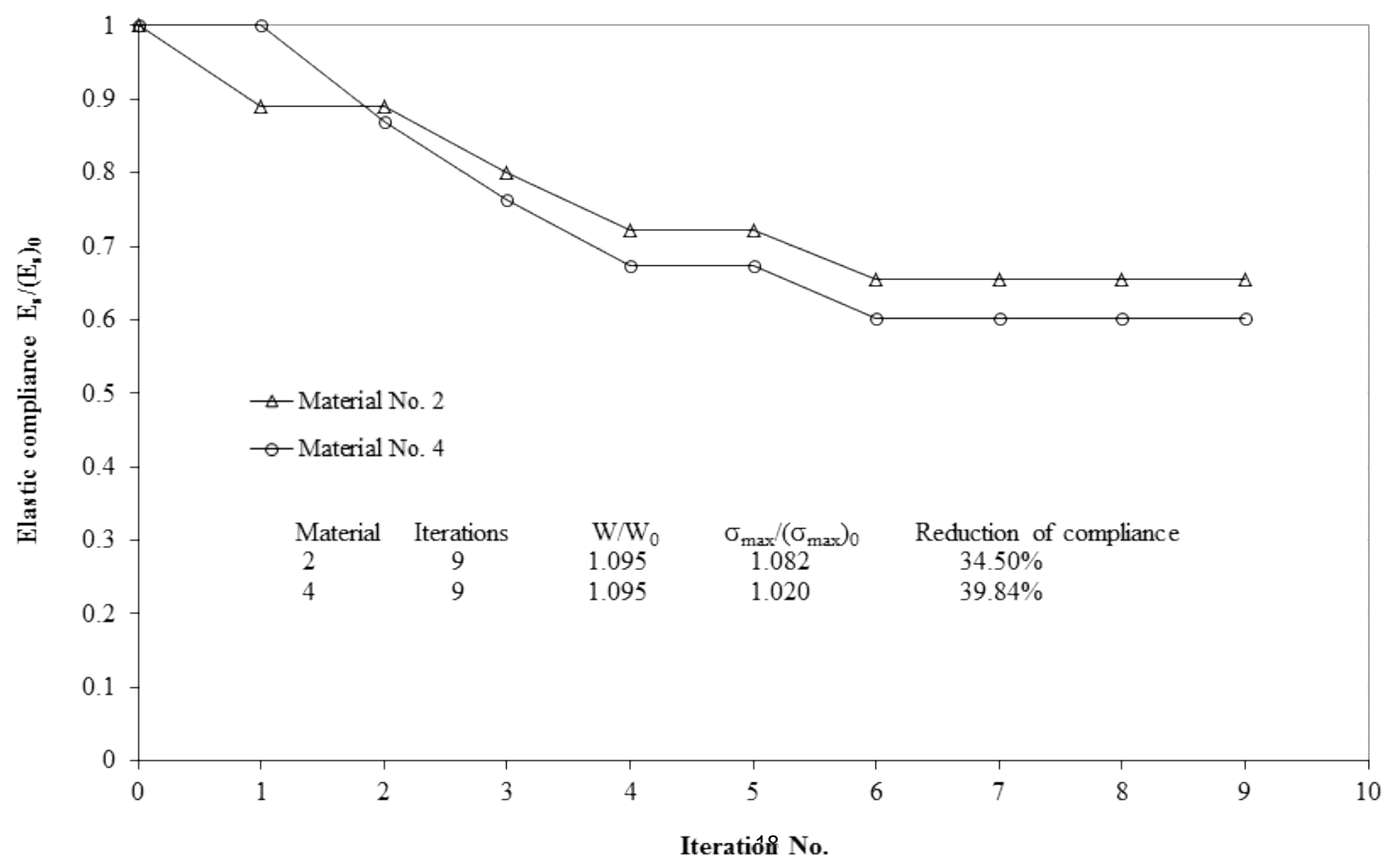

